\title{
Le remplacement de la canine en prothèse : analyse et rapport de cas cliniques
}

\section{RÉSUMÉ}

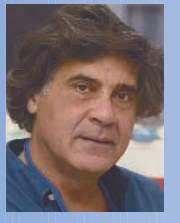

\section{Yves SAMAMA}

Docteur en Chirurgie Dentaire,

Docteur en Sciences Odontologiques,

Ex-assistant à la faculté

de chirurgie dentaire de Paris V,

3, place du 18 juin 1940,

75006 Paris.

\section{Philippe RAJZBAUM}

Docteur en Chirurgie Dentaire,

Docteur en Sciences Odontologiques,

Ex-assistant à la faculté

de chirurgie dentaire de Paris V,

57 , rue du président Wilson,

92300 Levallois.
Le dépistage désormais plus précoce des agénésies de canines et des canines incluses ainsi que les doléances esthétiques actuelles de patients plus âgés non traités, nous ont conduit à nous interroger sur l'opportunité des thérapeutiques prothétiques actuelles. Une réflexion sur les bridges collés et les constructions implantaires a été entreprise.

Cet article met en évidences qu'hormis l'analyse habituelle des critères cliniques indispensables à toute décision prothétique, il est utile de prendre en compte l'âge du patient ainsi que sa motivation en raison de la longueur et de la difficulté des traitements. L'option implantaire ne pourra être retenue de façon systématique dans tous les cas et la prothèse collée ou classique peut sembler la solution indiquée. Les difficultés esthétiques sont souvent très difficiles à évaluer en prothèse implanto-portée, entraînant des déceptions ou des échecs. Ces problèmes nous apparaissent souvent en rapport avec une mauvaise appréciation des difficultés dès lors qu'il s'agit d'édentement de nature et de type différent, associant des destructions tissulaires plus ou moins importantes.

Lorsque les conditions initiales ne sont pas favorables à une approche implantaire (espace coronaire réduit, défauts ostéomuqueux très importants) les efforts entrepris peuvent apparaître quelquefois démesurés; il semble intéressant d'évoquer la facilité que les restaurations prothétiques collées ou classiques peuvent apporter dans certains cas.

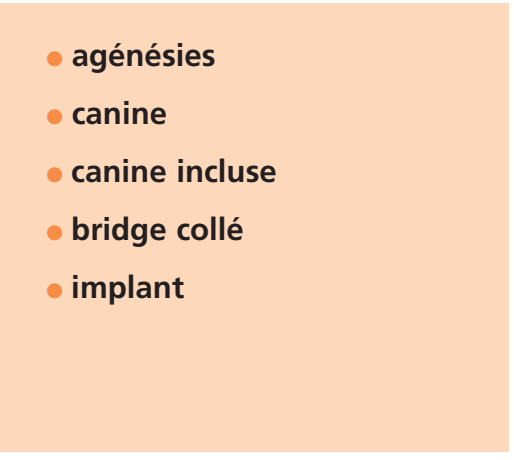

AOS 2008;244:317-344

DOI:10.1051/aos:2008041

(C) AEOS / EDP Sciences 


\section{Introduction}

En raison de sa situation sur l'arcade, de sa morphologie coronaire et radiculaire, le remplacement de la canine constitue un problème spécifique en odontologie. Par ailleurs, pendant plusieurs décennies, on a attribué à cette dent un rôle fonctionnel majeur alors qu'en réalité, le concept de protection canine est lié à des propositions thérapeutiques dans lesquelles le guidage canin intervient de façon plus ou moins importante.

D'un autre côté, la canine maxillaire participe au soutien de la lèvre supérieure au repos comme lors du sourire. L'absence de cette dent pose donc un problème esthétique et fonctionnel surtout lorsque l'on est confronté à un projet de réhabilitation prothétique important notamment lors de situations d'agénésies multiples ou d'accident traumatique sévère.

Quatre étiologies distinctes (en dehors des facteurs iatrogènes qui ne peuvent être abordés, car trop particuliers) expliquent l'absence de cette dent :

- l'ectopie ;

- le traumatisme qui est rarement, voire exceptionnellement, isolé ;

- l'agénésie simple (absence de germe définitif) ;

- les agénésies multiples (absence de plusieurs germes).
On pourrait croire, à I'heure de l'avènement de l'implantologie, que le remplacement de cette dent pourrait être simple. En fait, la nature de la réponse thérapeutique va être conditionnée par les étiologies évoquées car elles influencent de manière notable les conditions cliniques de son remplacement.

Nous nous proposons d'étudier ces différentes situations en évoquant des édentements de ce type, traités depuis le début des années 1980 , et en analysant les résultats obtenus au regard des possibilités actuelles (collage et implantologie).

En effet, durant ces deux dernières décennies, les solutions prothétiques non invasives se sont développées dans la pratique quotidienne en raison :

- d'une part, d'une meilleure compréhension dans les années 1980 des mécanismes interfaciaux et par l'amélioration des polymères de collage qui a permis la réactualisation des bridges collés décrits par Rochette en $1973[1,2,3,4,5,6,7]$;

- d'autre part grâce au concept d'ostéointégration introduit par Branemark (1977) qui a permis à l'implantologie de devenir une solution thérapeutique de choix pour traiter les édentements, y compris les édentements unitaires[8, 9, 10, 11, 12, 13, 14].

\section{L'ectopie de la canine}

Ce problème concerne surtout la canine maxillaire et survient dans environ $2 \%$ des cas (population européenne). La persistance de la canine temporaire en est le signe. La remise en place de la canine ectopique par l'orthodon- tiste devra être privilégié chez le patient jeune. Un diagnostic et un plan de traitement global en collaboration avec l'orthodontiste devront être envisagés. En effet, lors de l'ectopie de la canine maxillaire, il existe souvent des trou- 
bles associés comme des malpositions de dents adjacentes, des anomalies de forme des incisives latérales voire même des agénésies d'inci- sives latérales (I'agénésie de l'incisive latérale associée à une canine ectopique est relativement fréquente).

Exemple d'ectopie de la canine traitée par l'orthodontiste

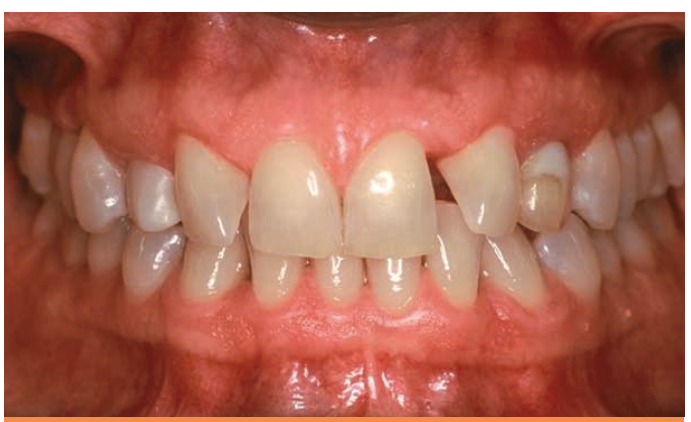

Fig. 1 Situation initiale : 13 et 23 sont incluses.

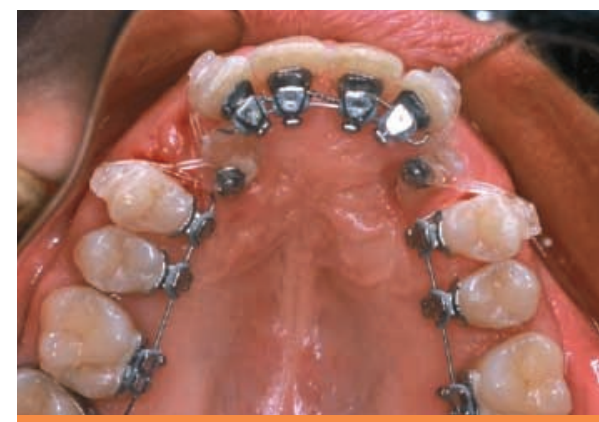

Fig. 3 Les canines sont dégagées chirurgicalement. Des boutons sont collés sur les faces palatines des canines, qui sont tractées par des élastiques.

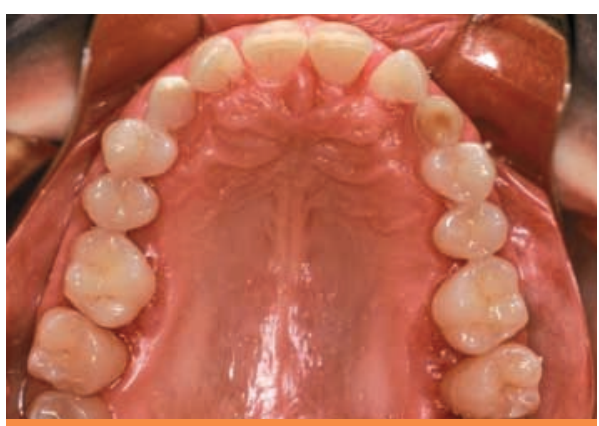

Fig. 2 Vue occlusale: 53 et 63 sont présentes sur l'arcade.

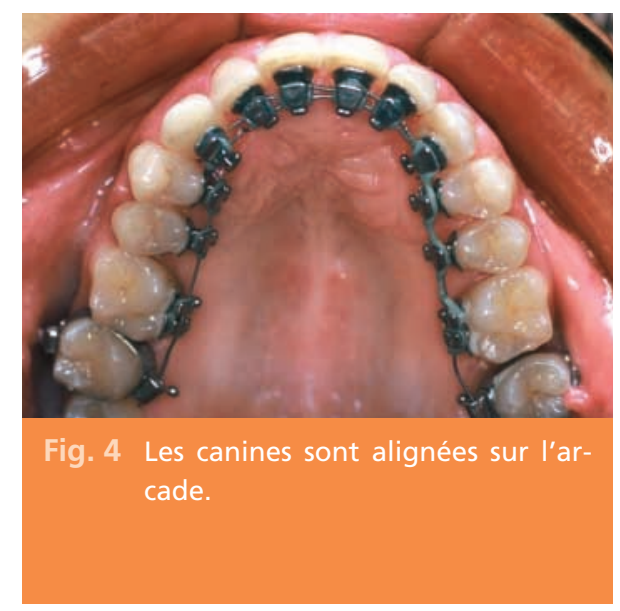

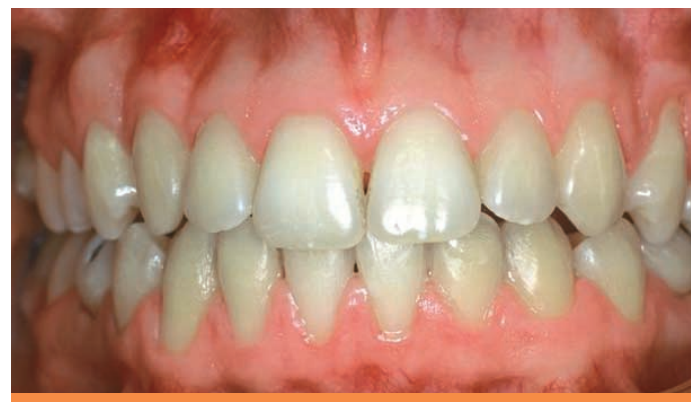

Fig. 5 Vue vestibulaire en fin de traitement. Traitement orthodontique : Eric Sarfati. 
Chez le patient âgé, la mise en œuvre d'un traitement orthodontique est souvent récusée. L'extraction de la canine incluse, l'éventuel comblement de la perte de substance occasionnée par cette extraction et la pose d'un implant s'avèrent souvent hors de propor-

\section{Le traumatisme}

L'absence de la canine peut être en rapport avec un choc traumatique; dans cette hypothèse, cette absence est rarement isolée et on observe souvent des conséquences parodontales. Plus généralement, la perte isolée de la canine est en rapport avec une manœuvre chirurgicale (extraction d'une canine incluse) ou approche chirurgico-orthopédique de remise en place tion. Dans la mesure où aucun signe clinique n'est présent, on s'orientera dans la plupart des cas vers l'abstention chirurgicale associée à une approche prothétique pure (bridge classique ou collé après perte de la canine lactéale). malheureuse. Ce type de traitement (remise en place chirurgicale) doit désormais rester exceptionnel compte tenu du dépistage précoce de la canine incluse et des progrès de l'orthodontie moderne. Dans notre pratique, il a été observé que cette approche conduit bien souvent à un échec avec une perte de substance importante voire à la perte de l'incisive latérale adjacente.

1 er exemple :

Perte de la canine en rapport avec une manœuvre

chirurgico-orthopédique

> Réalisation de préparations partielles sur les 2 prémolaires avec la canine en extension (cas traité en 1977)
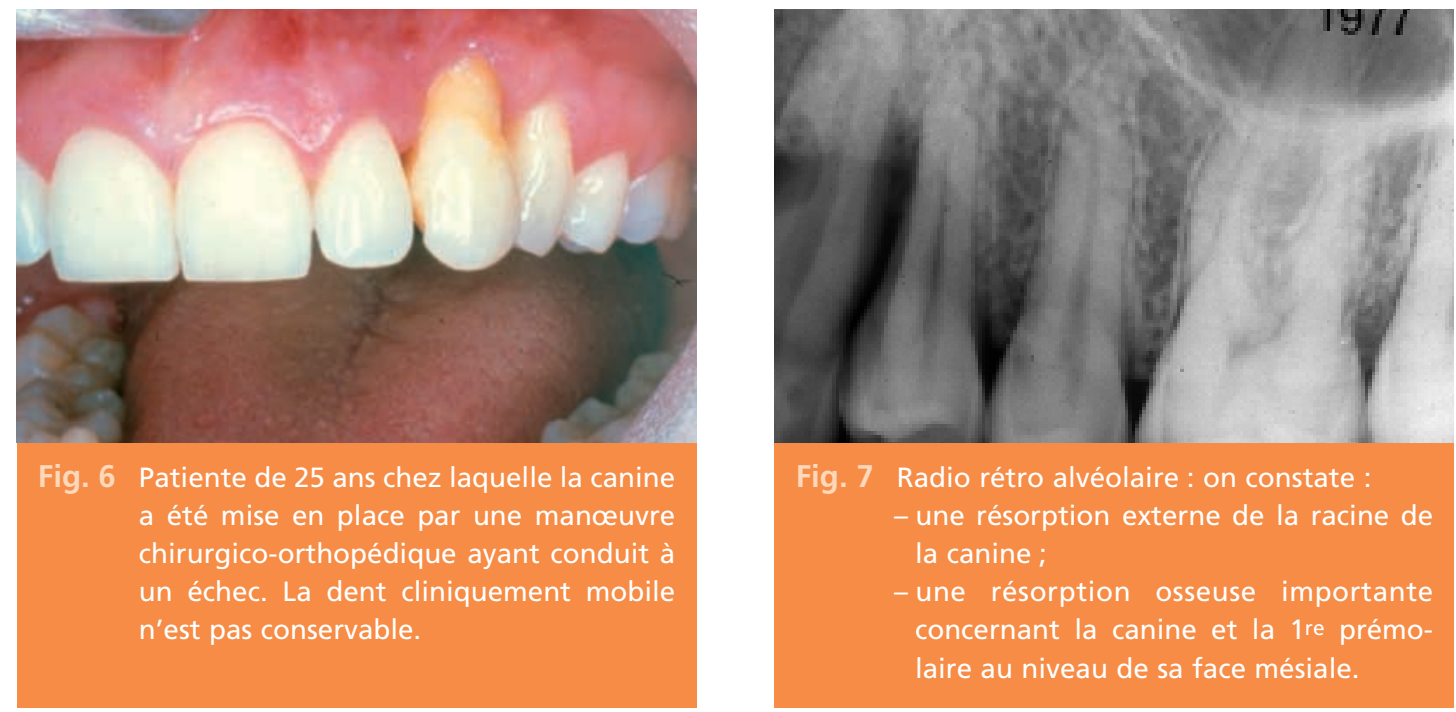
L'indication d'extraction de cette canine est sans ambiguïté. Ce type de situation aurait nécessité un tel niveau de complexité pour l'approche implantaire (reconstitution osseuse et implant) que le recours à une solution pro- thétique plus classique se pose. Par ailleurs, une reconstruction par greffe osseuse aurait pu conduire au «sacrifice» de la 1 re prémolaire afin de disposer d'un mur osseux apte à favoriser la réussite de la greffe osseuse.

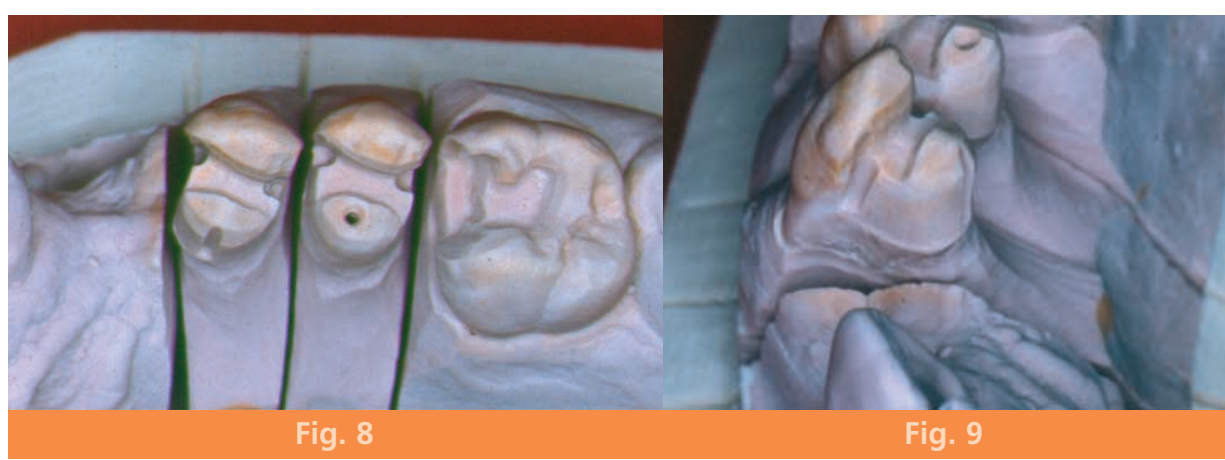

Fig. 8 et Fig. 9 Vue de la solution prothétique proposée : compte tenu de la petite taille de la latérale (faible indice de Le Huche) et dans le souci de l'économie tissulaire, 2 onlays ont été réalisés sur les 2 prémolaires, pour supporter une canine en extension ; les 2 onlays ont été scellés (le collage n'étant pas développé à l'époque). Actuellement, quasiment le même type de préparation serait proposé à l'exception de la vis palatine sur la $2^{e}$ prémolaire. En outre, les onlays seraient collés. Néanmoins, des rétentions macro mécaniques (rainures et tranchées) seraient encore tout autant nécessaires pour optimiser le pronostic de la construction.

Résultats cliniques après pose du dispositif prothétique :
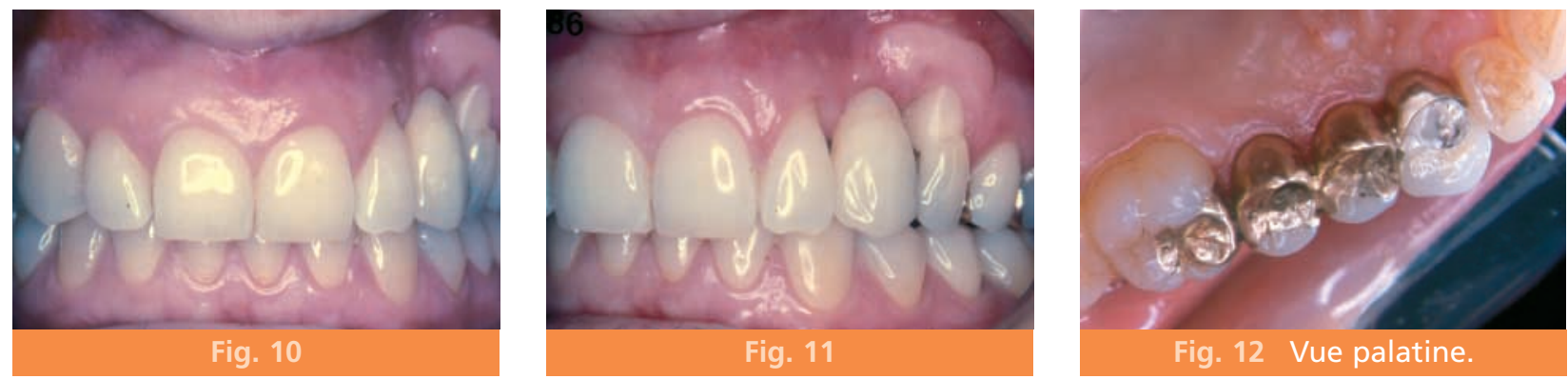

Fig. 10, Fig. 11 Vues vestibulaires.

Une greffe gingivale a été réalisée au niveau de la 1re prémolaire pour renforcer le parodonte. La perte de substance n'avait pas été traitée compte tenu des possibilités chirurgi- cales de l'époque (chirurgie R. Detienville). Aujourd'hui, le comblement de la perte de substance par un conjonctif enfoui s'imposerait. 


\section{- Analyse a posteriori :}

Les limites actuelles de la régénération osseuse dans le sens vertical constitueraient toujours une entrave à l'approche implantaire. En effet dans ce cas la racine de la prémolaire est exposée en grande partie dans le déficit osseux, ce qui impli- querait en cas de greffe osseuse l'extraction de cette dent. Cependant, si le volume osseux avait été suffisant en dépit de la perte osseuse, l'approche implantaire aurait été possible et le problème aurait été essentiellement esthétique, avec une couronne clinique très longue.

2e exemple :

Perte de la canine associée à celle de la latérale avec mésialisation partielle du groupe cuspidé. Approche classique scellée (cas traité en 1983-1984)
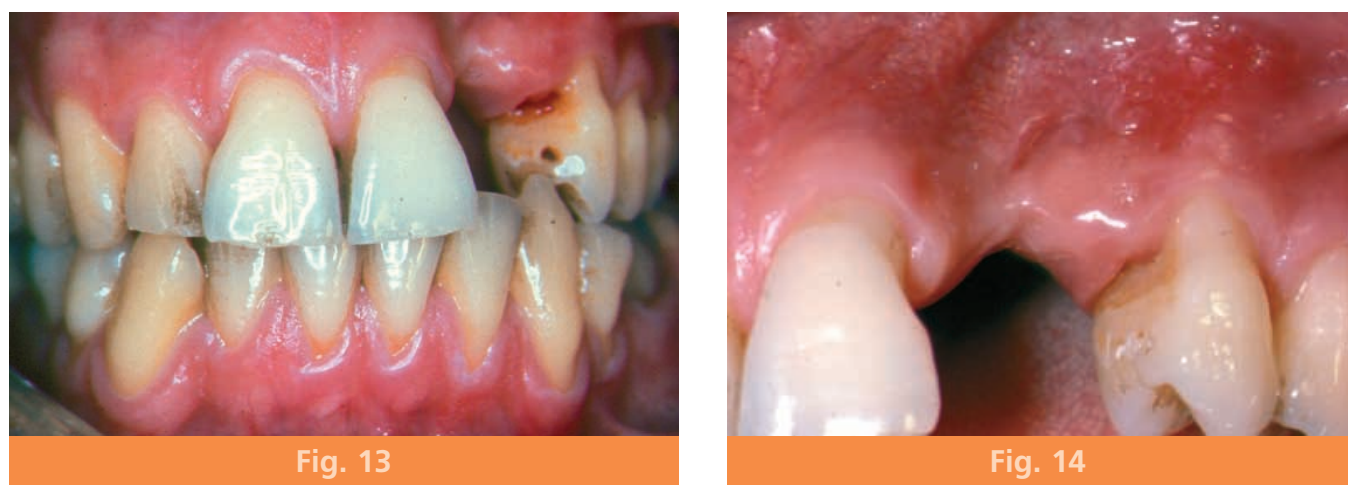

\section{Fig. 13 et Fig. 14 Vues préopératoires:}

on note l'absence de la canine et de l'incisive latérale associée à une grosse perte de substance ostéo muqueuse.

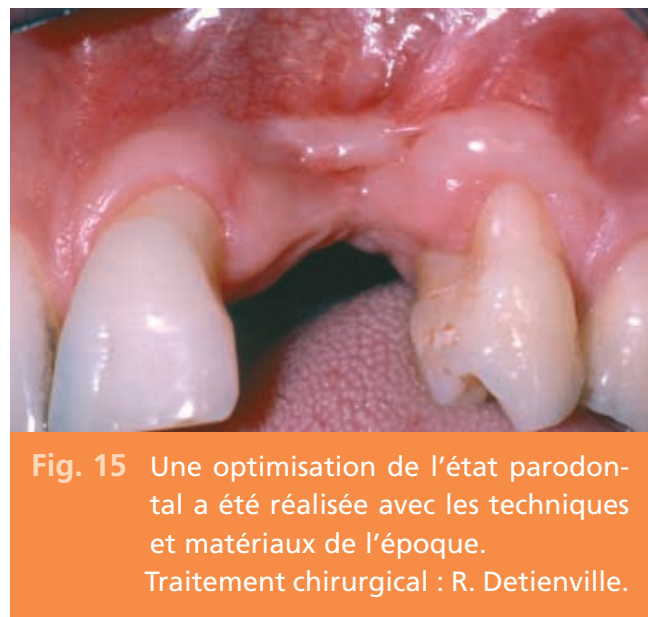



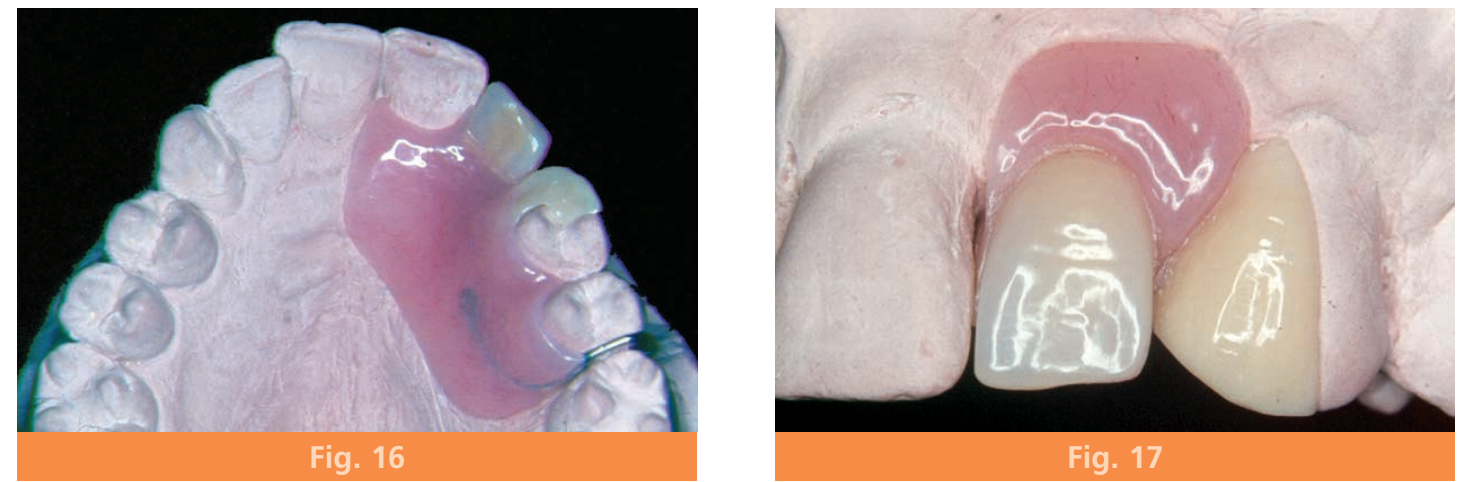

Fig. 16 et fig. 17 Simulation prothétique avec une PAP : on note qu'il est impossible de remplacer la canine et l'incisive latérale compte tenu de la mésialisation du groupe cuspidé. Il a été décidé de réaliser une couronne périphérique surdimensionnée sur la prémolaire en lui donnant un aspect vestibulaire en forme de canine et deux onlays sur les incisives centrales.
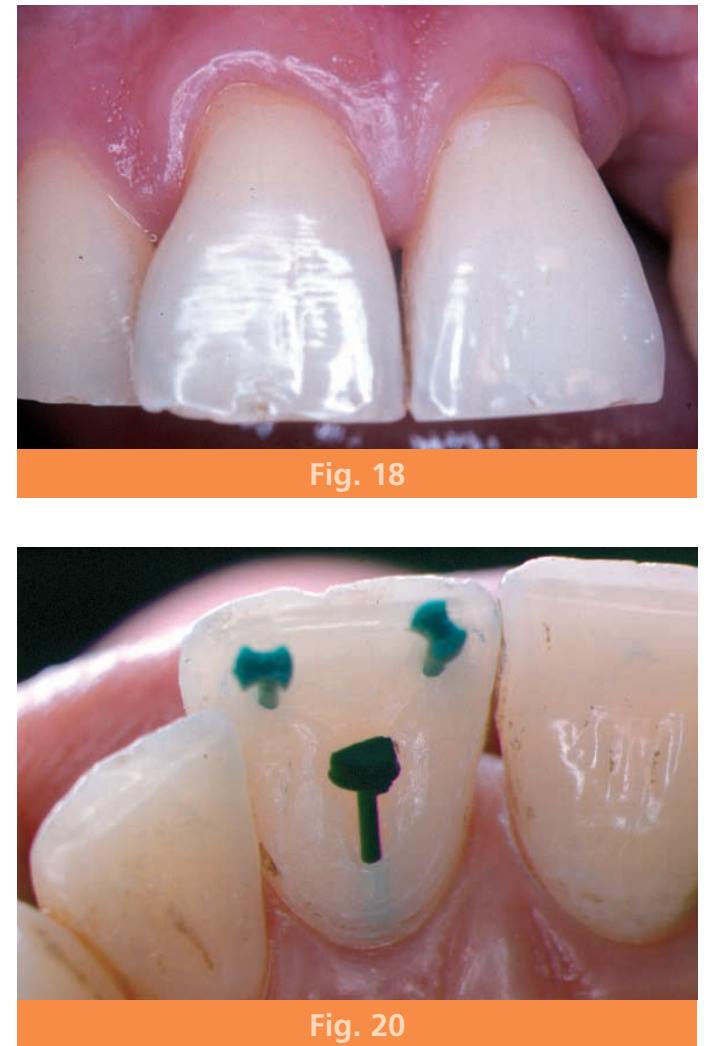

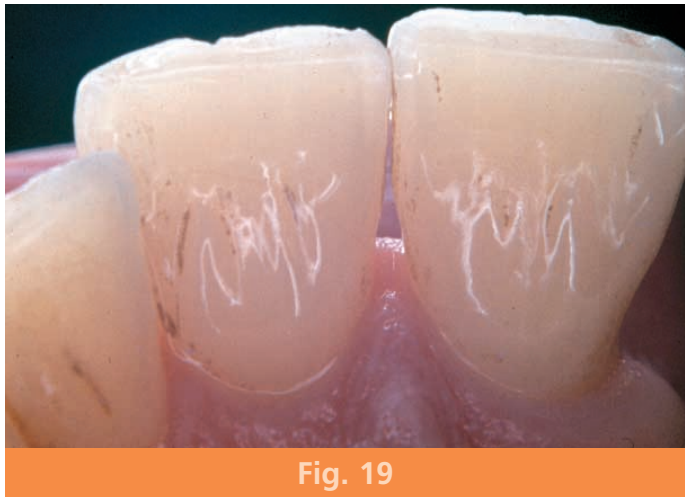

Fig. 18, Fig. 19 et Fig. 20

Les incisives centrales sont très massives et triangulaires : elles se prêtent parfaitement à la réalisation d'onlays surtout en raison de leur forme triangulaire. À l'époque, des onlays en Y ont été réalisés (décrits par les Dr Brunnel, Lackermans et Leibowitch). 

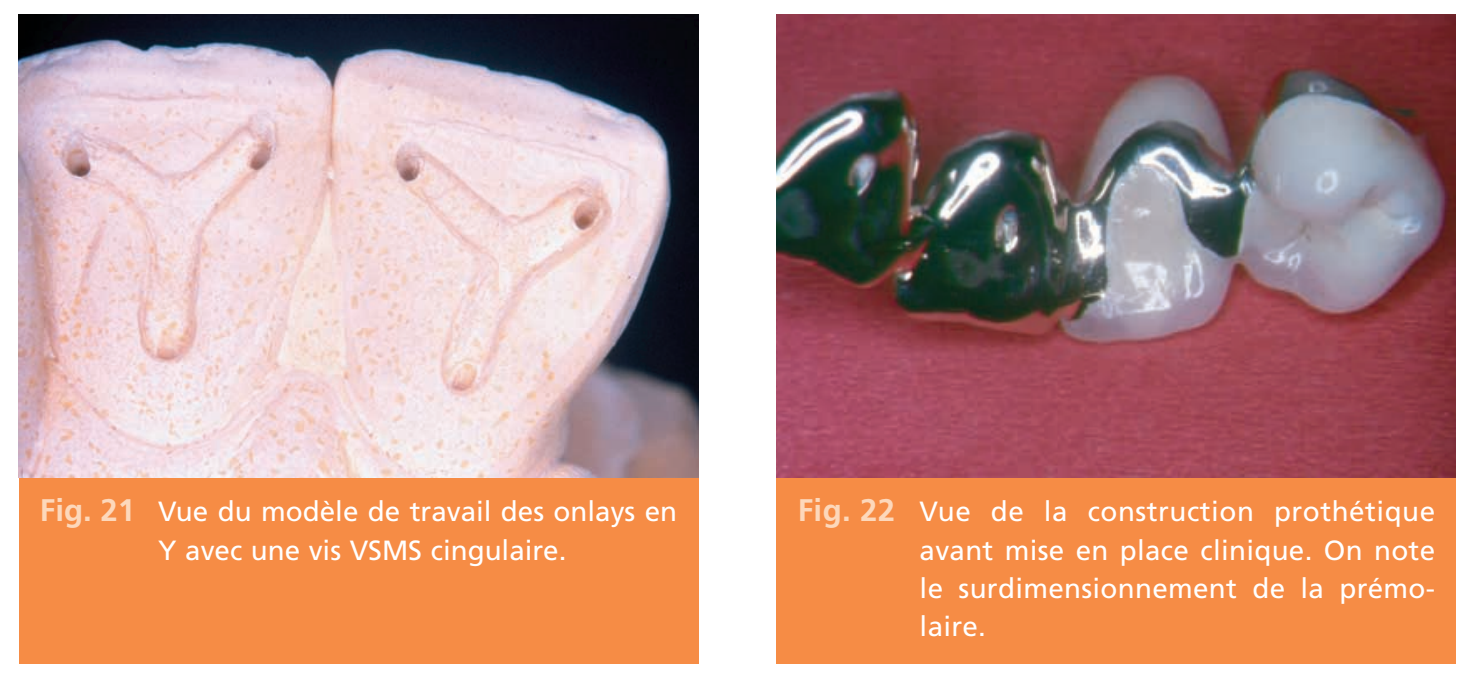

Fig. 22 Vue de la construction prothétique avant mise en place clinique. On note le surdimensionnement de la prémolaire.
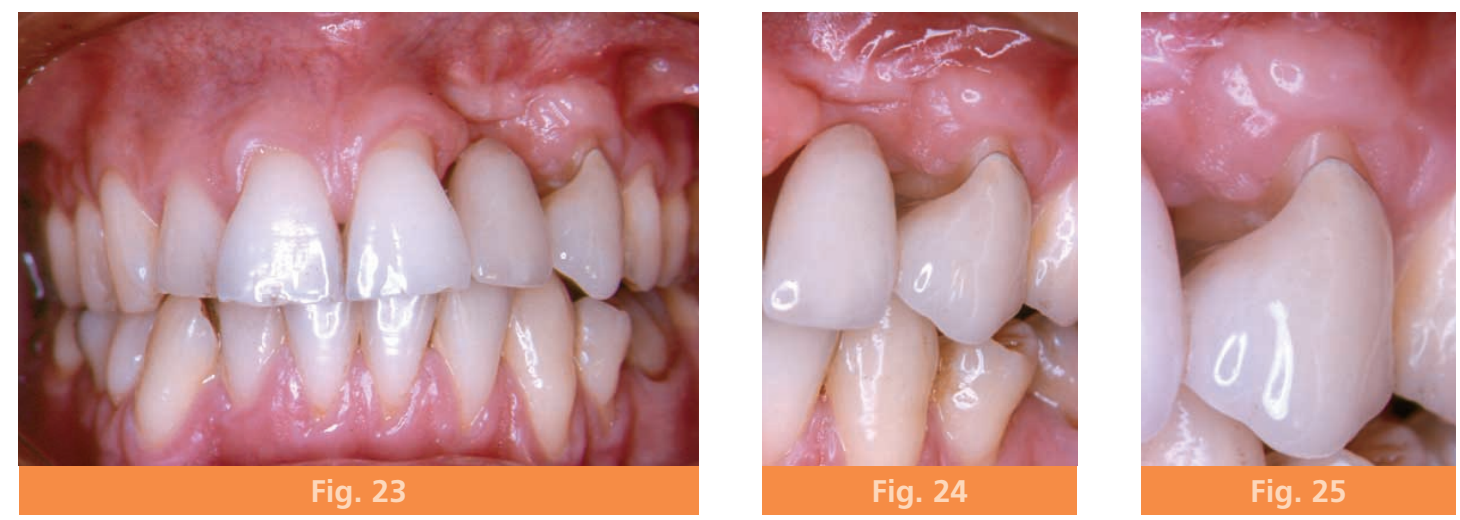

Fig. 23, Fig. 24 et Fig. 25

Vues cliniques postopératoires. On note l'aspect vestibulaire de la prémolaire transformée en canine.

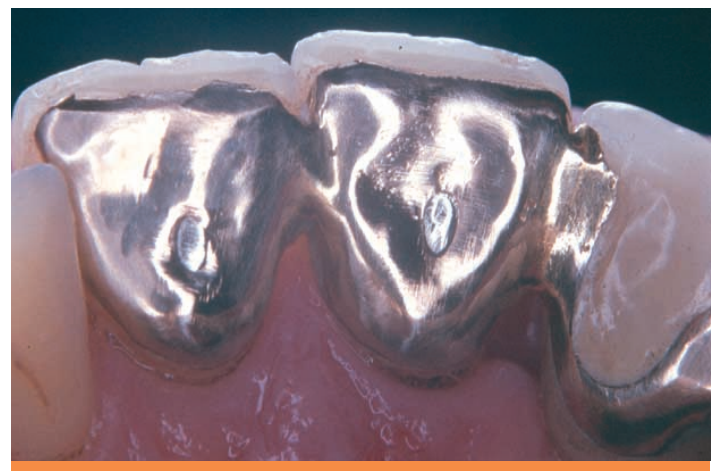

Fig. 26 Vue linguale des onlays après section des SMS. 

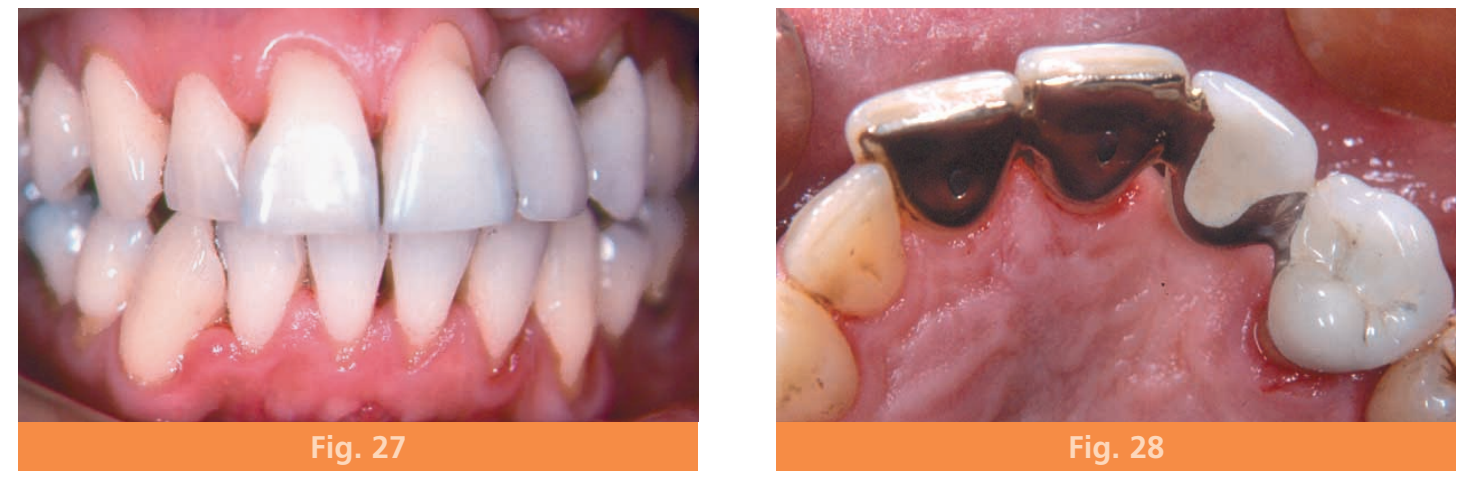

Fig. 27 et Fig. 28

Vues cliniques de la construction en 1999/2000, soit 14 ans postopératoire.

\section{- Analyse a posteriori :}

Même aujourd'hui l'option implantaire serait très difficile à envisager en raison d'une part de l'importance de la perte de substance, d'autre part de l'espace insuffisant entre 11 et 14 pour placer 2 implants. La perte de sub- stance pourrait être mieux compensée par un conjonctif enfoui. La forme de contour des onlays aurait été simplifiée en raison de l'apport de l'adhésion qui permet de réduire les rétentions macroscopiques et la profondeur des encastrements.

3e exemple :

Réalisation d'un bridge céramo-métallique destiné à remplacer la canine et la latérale.

Cas traité en 1986 avec des onlays collés

> Cas réalisé en 1986 et témoignant de l'apport du collage et des progrès réalisés dans le traitement de perte de substance.
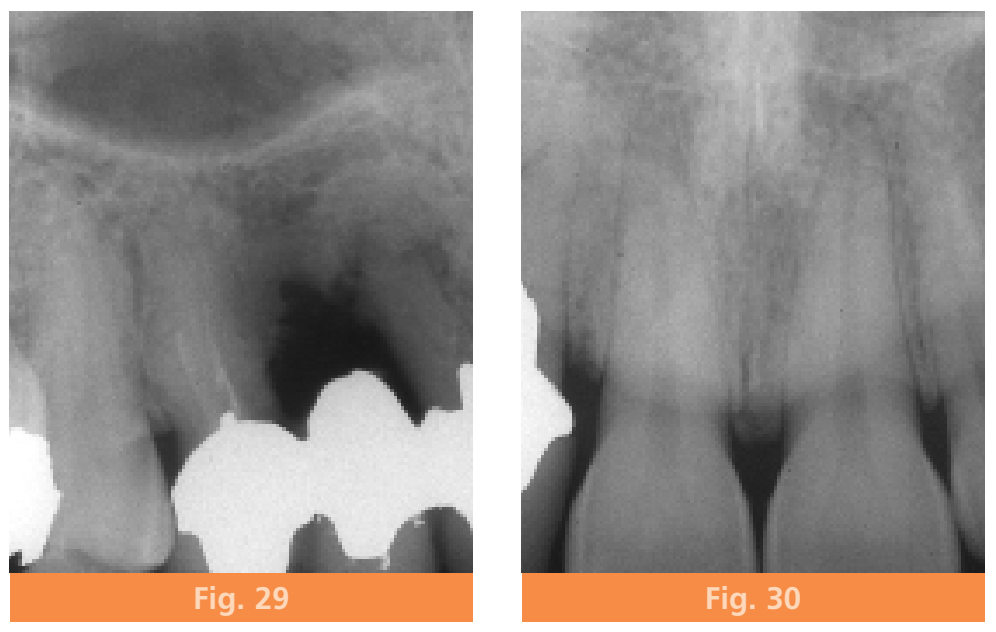

Fig. 29 et Fig. 30

Radios pré-opératoire : La canine absente avait été remplacée par un bridge céramo-métallique. L'incisive latérale est perdue et sera extraite ; la prémolaire sera retraitée et conservée ; sur les incisives centrales massives et triangulaires deux onlays collés seront réalisés. 


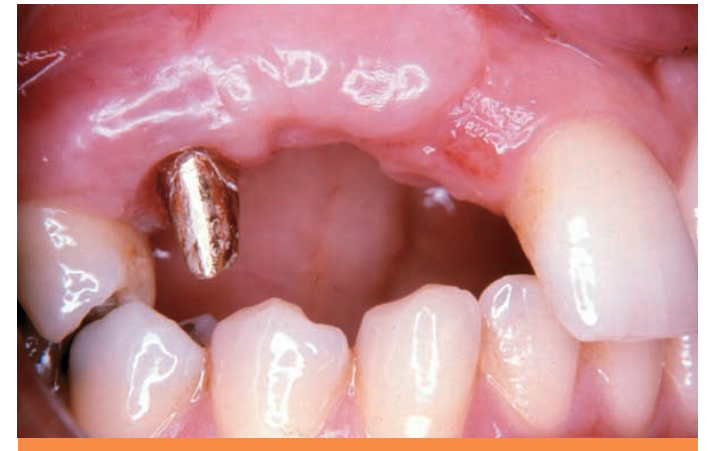

Fig. 31 Vue clinique peropératoire. Un conjonctif enfoui et une greffe épithélio conjonctive épaisse ont été réalisés pour réduire la perte de substance (Dr Laviec).
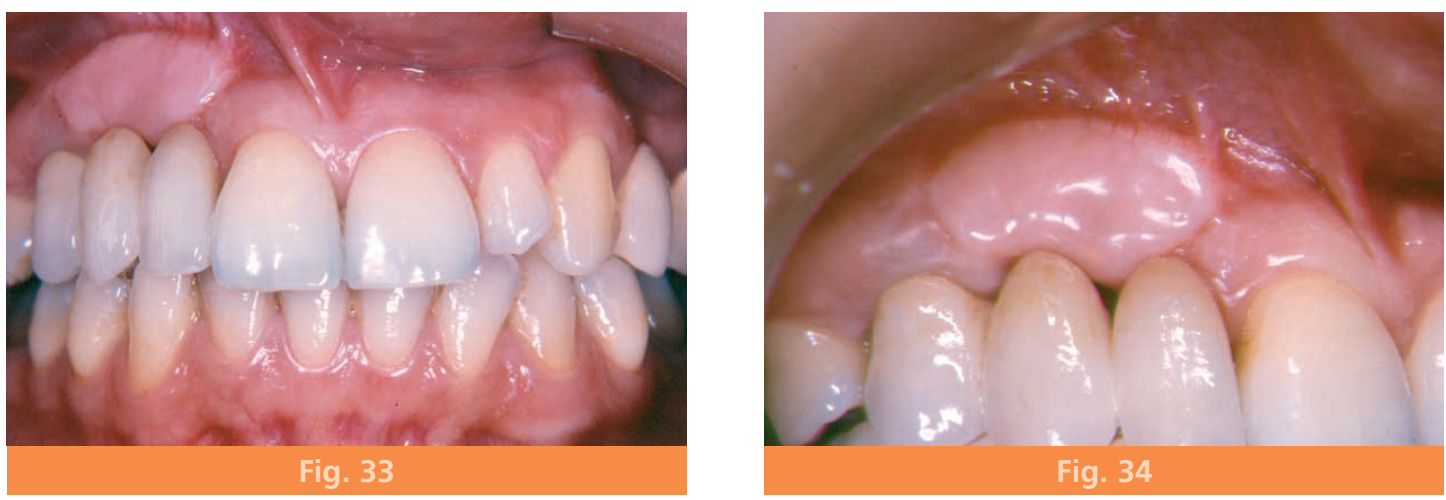

Fig. 33 et Fig. 34

Vues cliniques postopératoires. Les progrès en parodontie ont permis une meilleure compensation de la perte de substance, par du conjonctif enfoui.

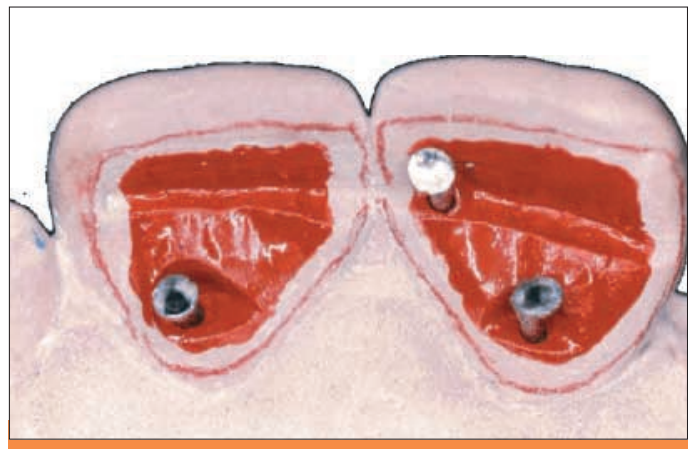

Fig. 32 On note une simplification de préparation par rapport au cas précédent grâce à l'exploitation de l'adhésion. Les profondeurs d'encastrement sont réduites ainsi que le nombre de tenons dentinaires.

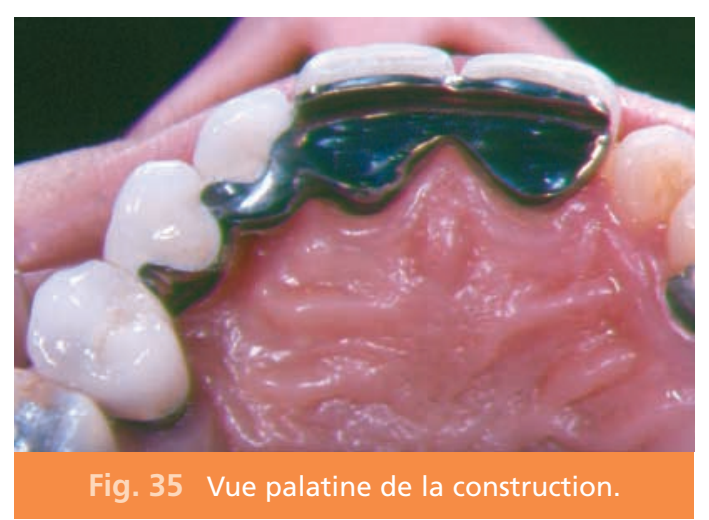

326 
4 e exemple :

Réalisation d'un bridge céramo-métallique pour le remplacement de la canine associée à la perte de la latérale

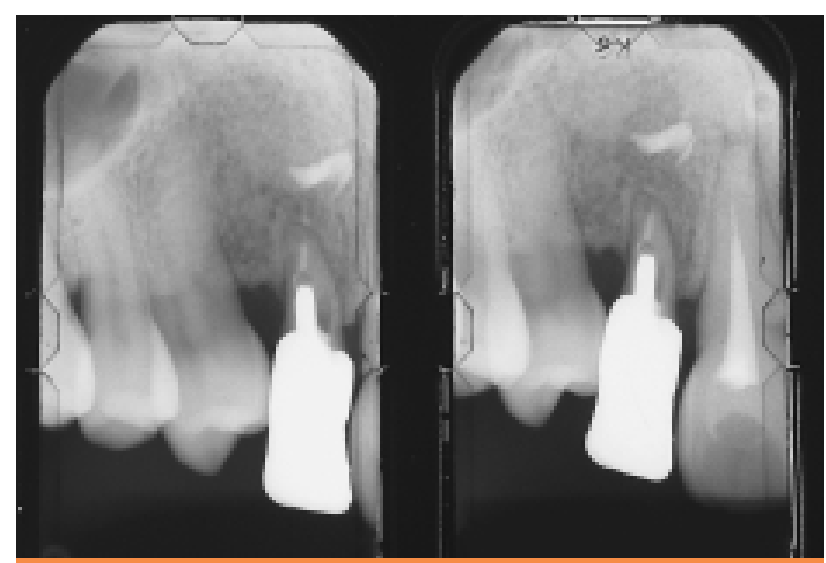

Fig. 36 Radio préopératoire attestant de la perte de la canine et du délabrement de l'incisive centrale dont le retraitement s'était avéré impossible. Une obturation à rétro sera réalisée (1982). On note une mésialisation de la prémolaire et la nécessité d'extraire l'incisive latérale.

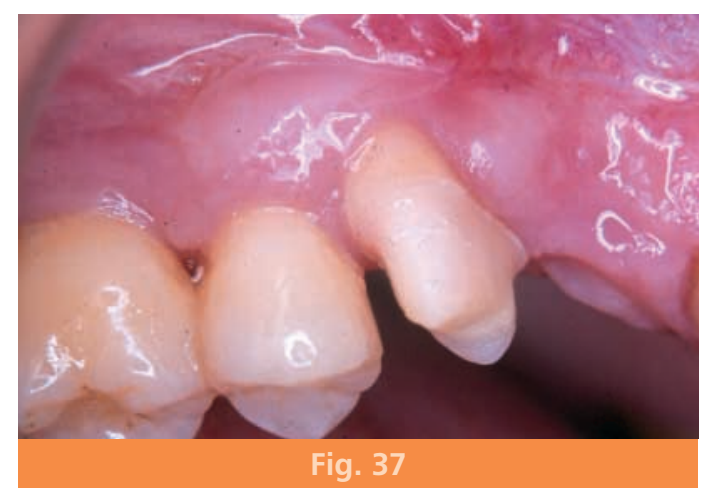

Fig. 37, Fig. 38 et Fig. 39

Vue de la préparation de la prémolaire transformée en canine avec une limite largement supra gingivale. La vitalité pulpaire a été conservée.
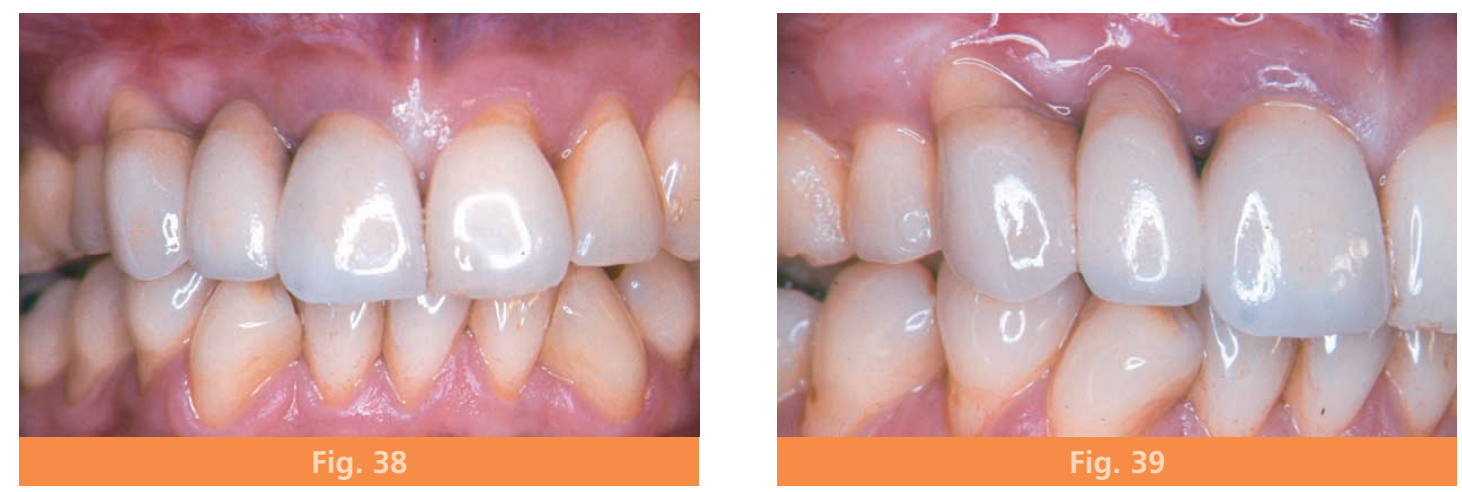


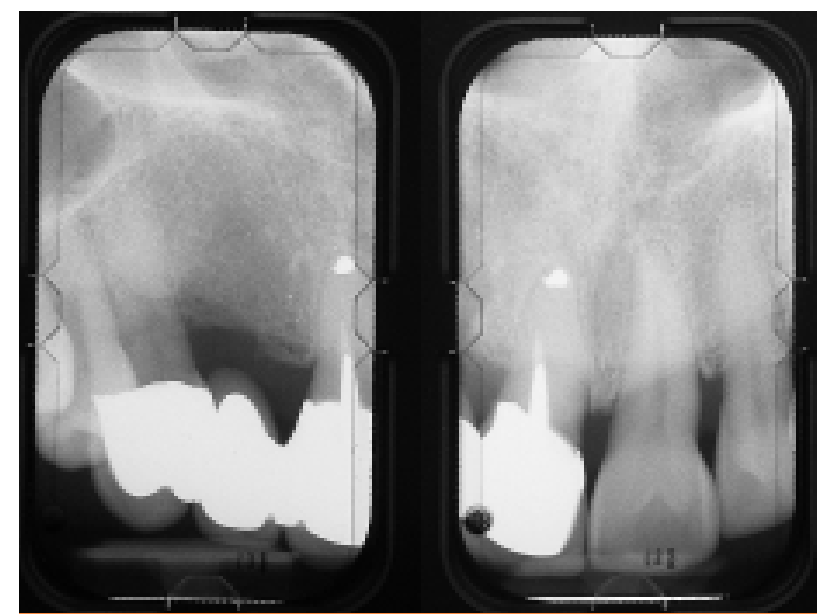

Fig. 40 Radiographie post-opératoire en 2007.

5e exemple :

Absence de canine en raison d'une fracture radiculaire isolée

\section{> L'option implantaire}

Chirurgicalement, l'avulsion de la racine résiduelle doit être menée avec précaution de manière à préserver la table osseuse vestibulaire ; on privilégiera la technique de fragmentation de la racine décrite par J.P. Gardella[15]. L'utilisation éventuelle de matériaux de com- blement foulés dans l'alvéole afin d'optimiser la cicatrisation osseuse est possible.

Lorsque l'évaluation du volume osseux est positive, un implant sera mis en place et la prothèse est réalisée avec une mise en charge immédiate ou différée selon les circonstances cliniques.

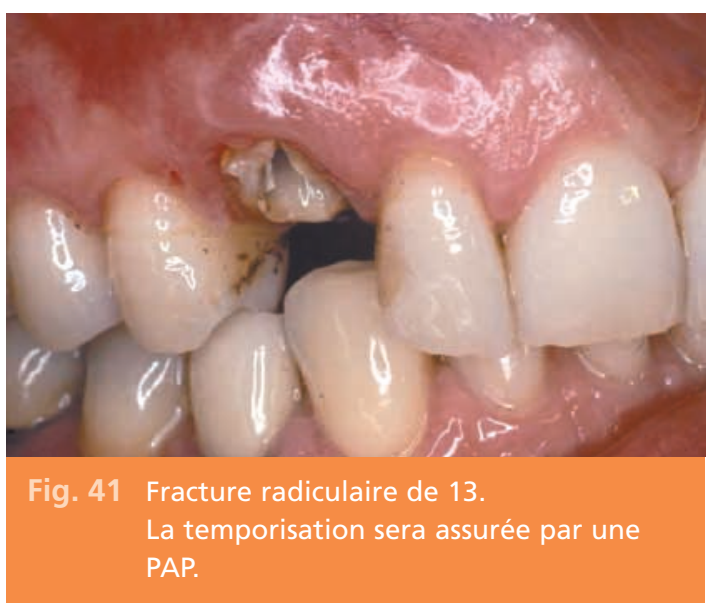



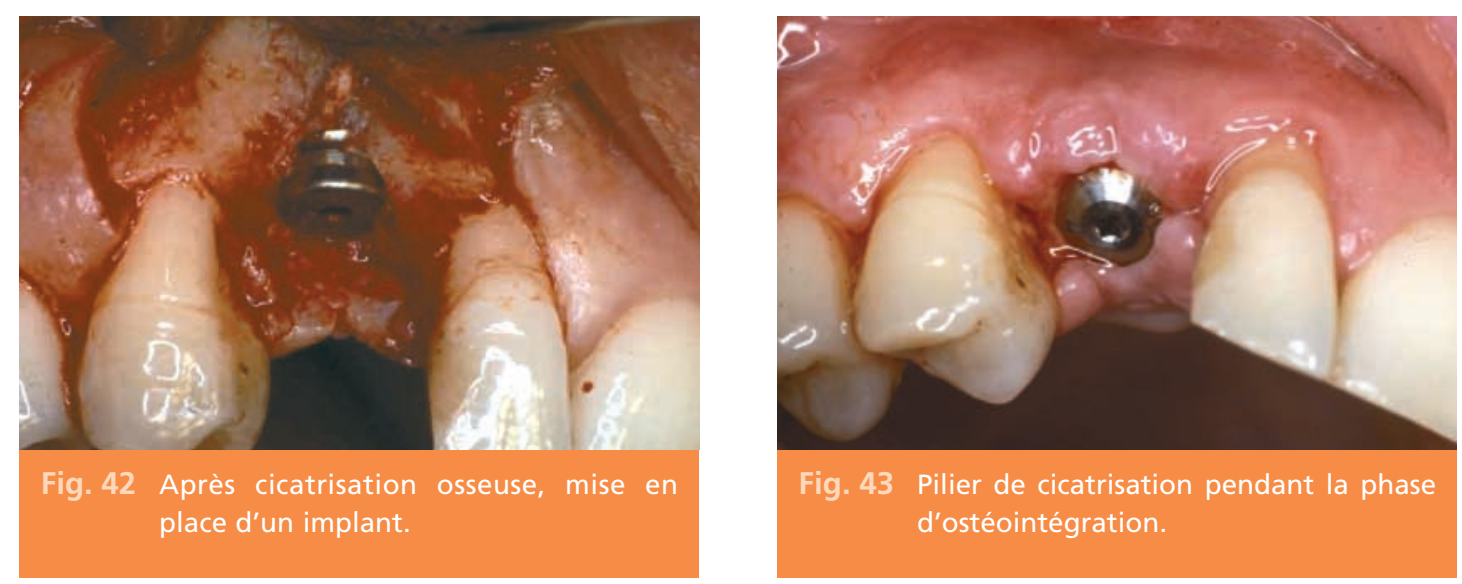

Fig. 43 Pilier de cicatrisation pendant la phase d'ostéointégration.
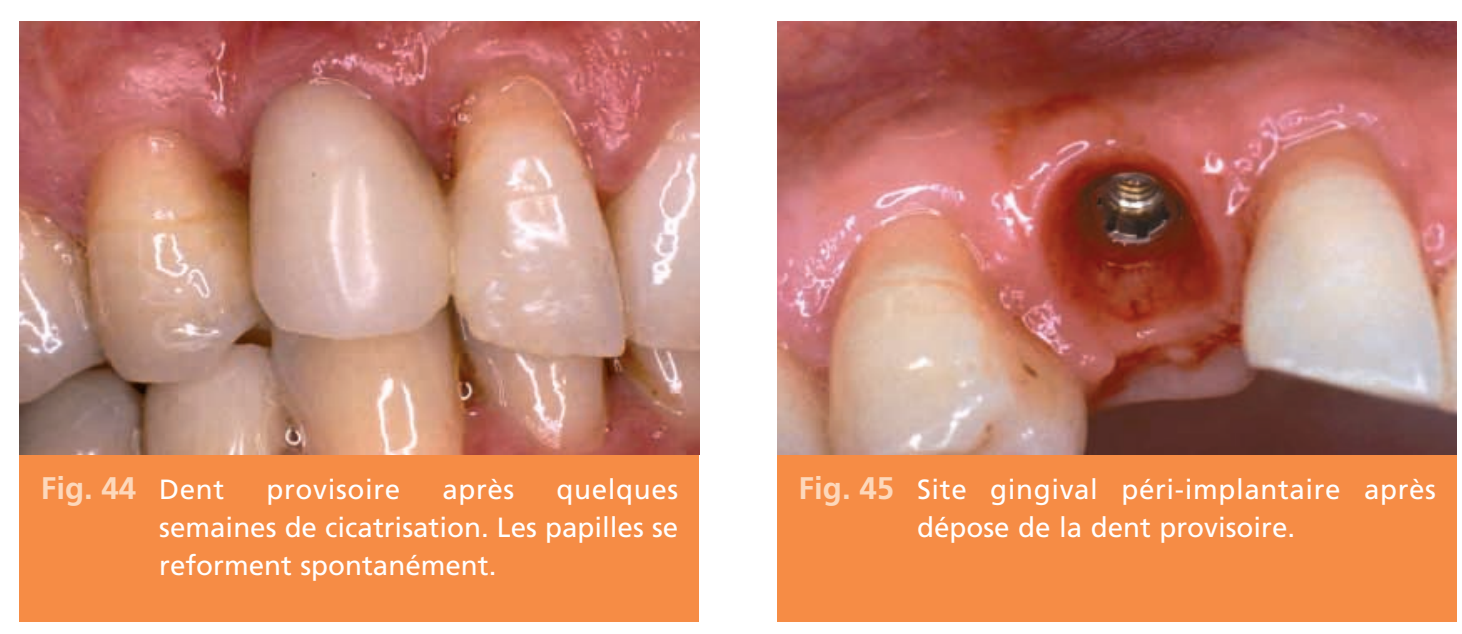

Fig. 45 Site gingival péri-implantaire après dépose de la dent provisoire.

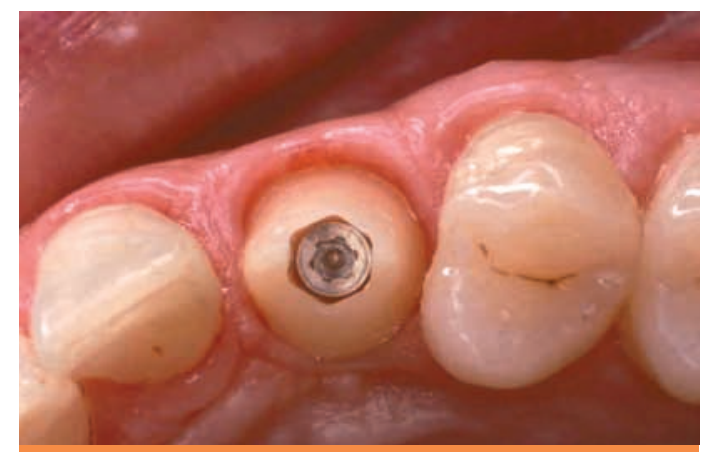

Fig. 46 Les limites cervicales du composant en alumine sont légèrement sous gingivales.

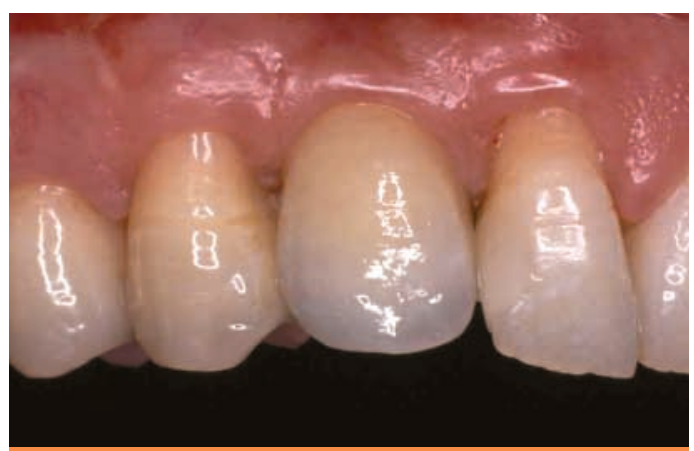

Fig. 47 Mise en place du pilier qui est vissé sur l'implant. La couronne céramo-céramique est scellée sur le pilier. Les mouvements occlusaux en latéralité sont réglés en fonction de groupe. 


\section{Agénésie isolée de la canine}

L'absence isolée du germe de la canine permanente est rare. La prévalence de l'agénésie de ce type de dent est résumée dans le tableau I[16]. Elle est nettement moins fréquente que les agénésies des incisives latérales et des prémolaires[17]. Comme bien souvent dans les cas d'agénésie, il existe une hypoplasie osseuse associée qui peut compliquer l'approche implantaire. On peut également se trouver (comme souvent dans les cas d'agénésie) confronté a un espace coronaire réduit qui empêche la pose d'un implant. Le bridge d'onlay permet d'assurer le remplacement esthétique et fonctionnel de la canine car généralement la population atteinte d'agénésie est exempte de lésions dentaires. C'est l'approche purement prothétique la moins invasive possible.

\section{Tableau I}

Résumant la prévalence des agénésies par type de dent (échantillon de 48274 sujets établis à partir de 10 études). D'après la méta-analyse de Polder et coll 2004[16].

\begin{tabular}{lllcc}
\hline & \multicolumn{2}{l}{ Maxillaire } & \multicolumn{2}{c}{ Mandibule } \\
\hline I1 & $n$ & $\begin{array}{c}\text { Prévalence } \\
(\text { IC } 95 \%)\end{array}$ & $\begin{array}{c}\text { Prévalence } \\
\text { (IC 95 \%) }\end{array}$ \\
I2 & 3 & $0,00-0,01$ & 143 & $0,25-0,35$ \\
C & 803 & $1,55-1,78$ & 102 & $0,17-0,25$ \\
P1 & 47 & $0,07-0,13$ & 8 & $0,01-0,03$ \\
P2 & 100 & $0,17-0,25$ & 66 & $0,10-0,17$ \\
M1 & 722 & $1,39-1,61$ & 1479 & $2,91-3,22$ \\
M2 & 17 & $0,02-0,05$ & 6 & $? ? ? 0,00-0,02$ \\
\hline
\end{tabular}

\section{Analyse de l'option bridge d'onlay}

Au niveau du bridge collé 2 options sont possibles et dépendront de l'examen clinique préalable :

- soit la préparation des deux prémolaires avec l'intermédiaire en extension ;

- soit la préparation de la prémolaire et de l'incisive latérale.
L'analyse de la forme des dents et la présence d'obturation existantes seront les facteurs décisionnels. Par ailleurs, I'amélioration des traitements de surface ainsi que celle des polymères de collage ont permis de simplifier les préparations, de les rendre plus accessibles avec des résultats probants. 


\section{>1re option:}

réalisation de préparations partielles sur les deux prémolaires

avec la canine en extension

\section{Cas réalisé en 1977.}

Patiente de 22 ans présentant une agénésie de la canine. La canine lactéale est encore présente sur I'arcade.

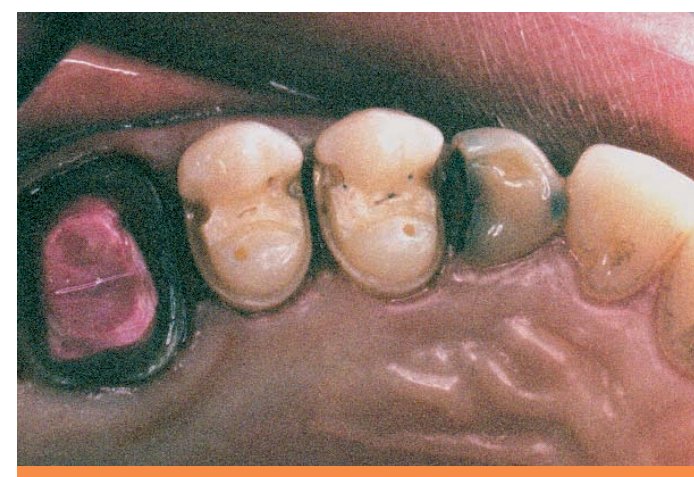

Fig. 482 onlays à rainures ont été réalisés afin de supporter une canine en extension. La rétention de ces onlays avait été majorée à l'époque par des vis VSMS. La dent lactéale sera extraite lors de la mise en place du bridge. L'éviction gingivale a été réalisée au bistouri électrique pour permettre une empreinte aux hydrocolloides.

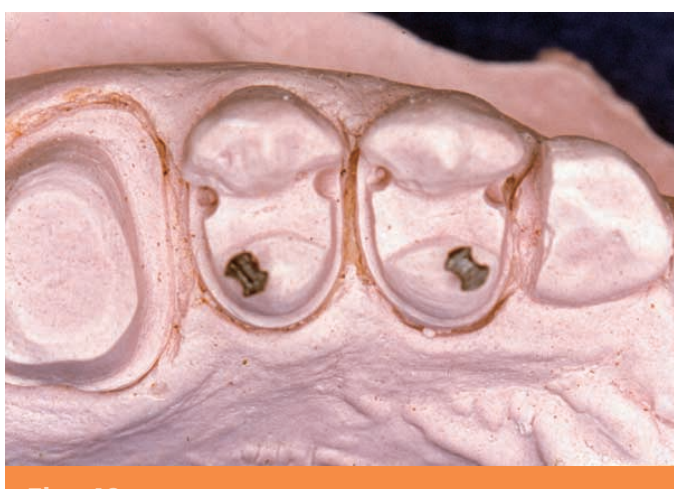

Fig. 49 Vue des préparations sur le modèle de travail. On note les éléments secondaires de rétention (rainures et vis VSMS sur les cuspides palatines).

Vue clinique du bridge mis en place le jour de l'extraction de la canine lactéale.

Un alliage nickel chrome avait été choisi à l'époque en raison de sa rigidité sous faible épaisseur.

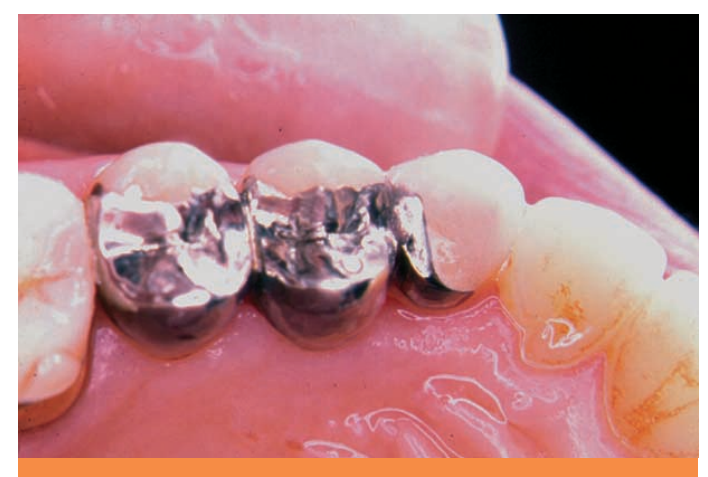

Fig. 50 Face palatine.

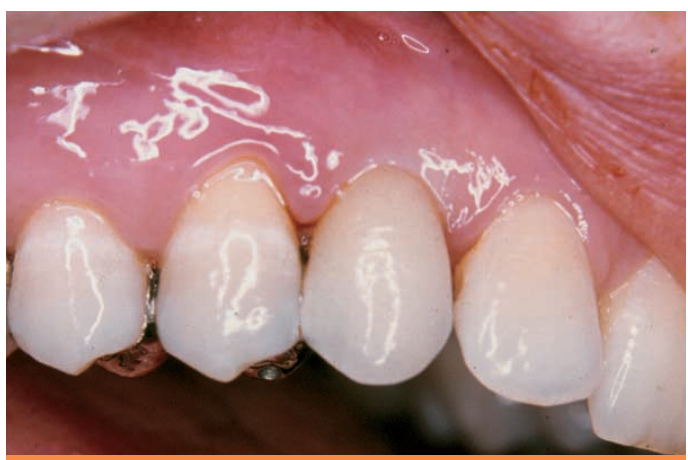

Fig. 51 Face vestibulaire le jour de la mise en place. 

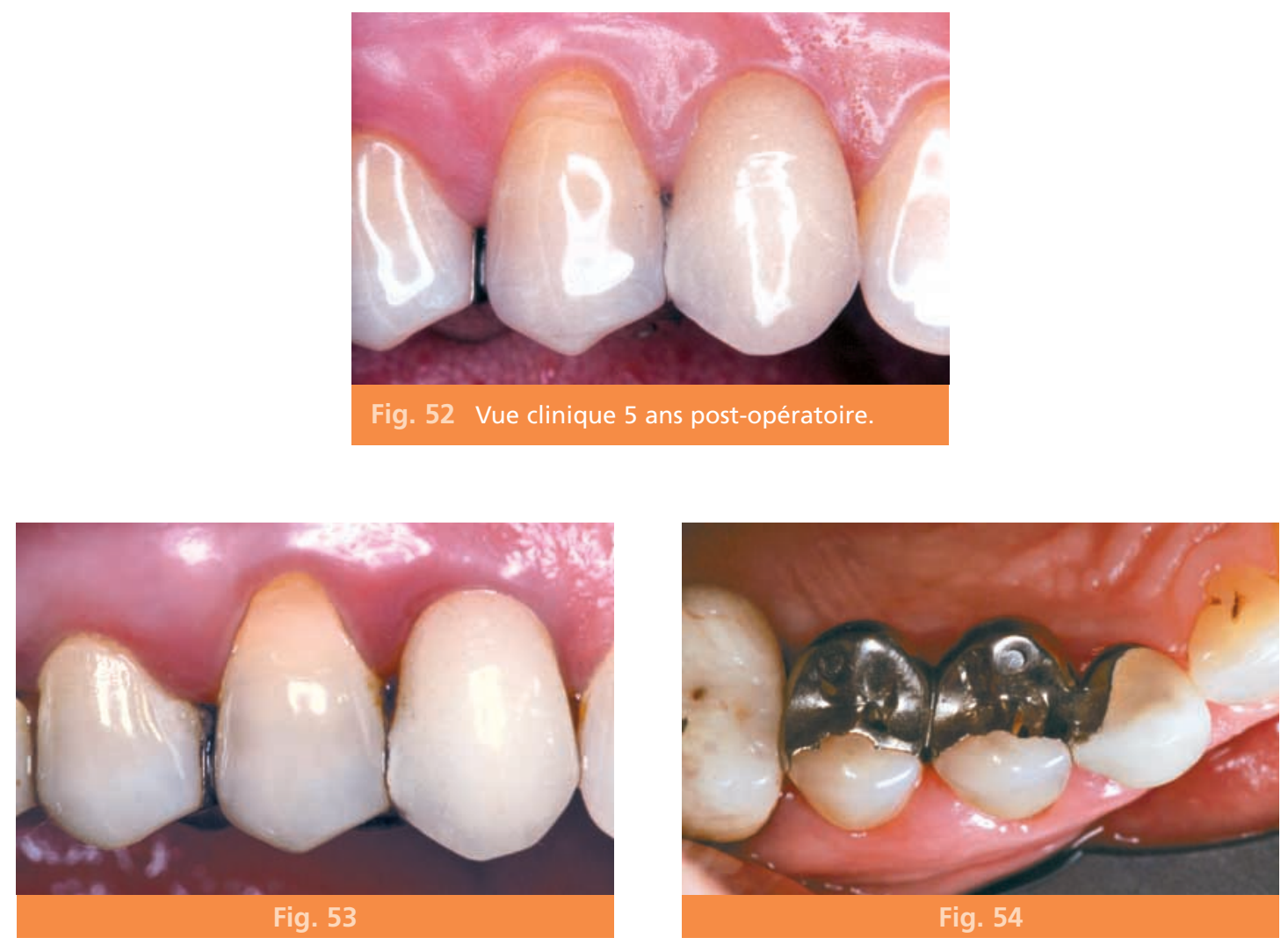

Fig. 53 et Fig. 54 Vues cliniques 30 ans postopératoires. Réalisation prothétique : J. Ollier.

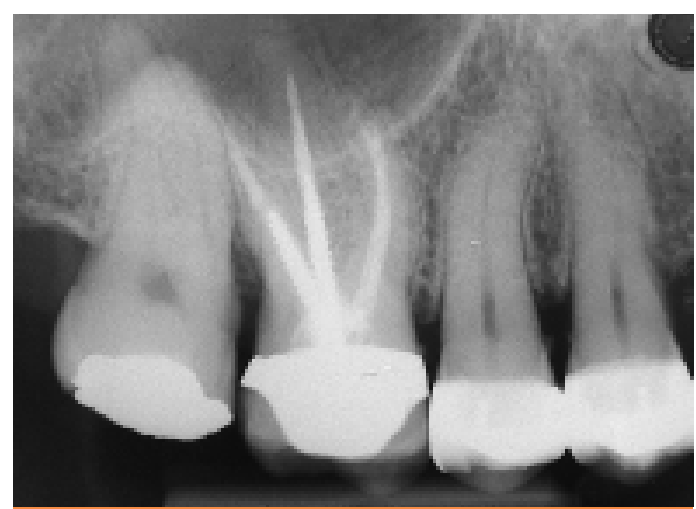

Fig. 55 Radio rétro alvéolaire 30 ans postopératoire.

\section{- Analyse a posteriori :}

Les formes de contour seraient plus superficielles mais voisines. On éviterait les vis VSMS.
La résine 4 méta TBB (Superbond) serait choisie pour un collage. 


\section{$>2^{\mathrm{e}}$ option :}

préparations sur la prémolaire et sur l'incisive latérale

\section{Cas réalisé en 1989}

Après collage, on note que dans le domaine esthétique, le résultat est plus prévisible avec un bridge collé.
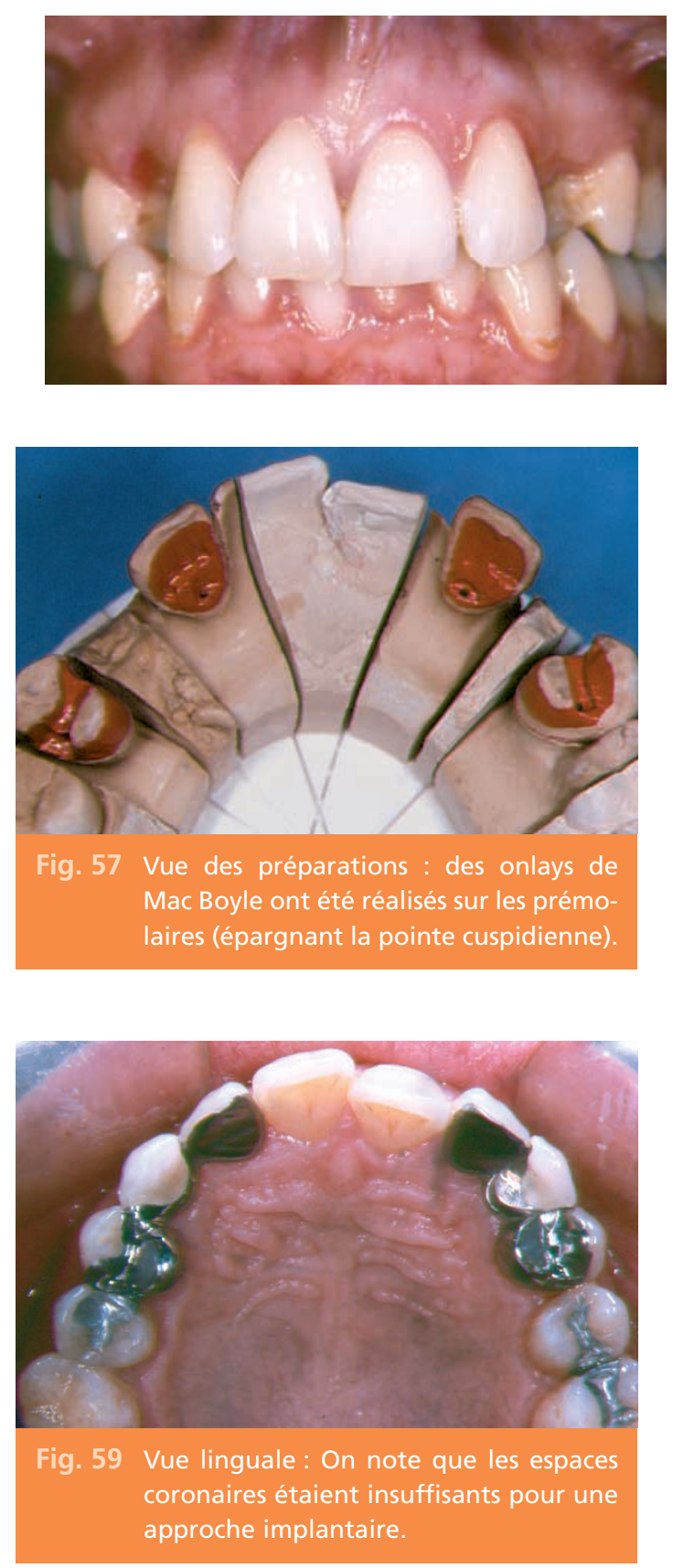

\section{- Analyse a posteriori :}

On choisirait une colle opaque (ivory opage du 4 méta TBB - Superbond) pour éviter l'interférence décolorante (gris au niveau du bord libre du métal).

Fig. 56 Vue clinique préopératoire chez une patiente présentant l'absence des 2 canines maxillaires. La temporisation a été établie dès l'adolescence par une PAP. La forme et la valeur des latérales se prêtent à la réalisation d'onlays collés. L'option implantaire n'était pas possible en raison de l'espace coronaire réduit et de la convergence radiculaire.

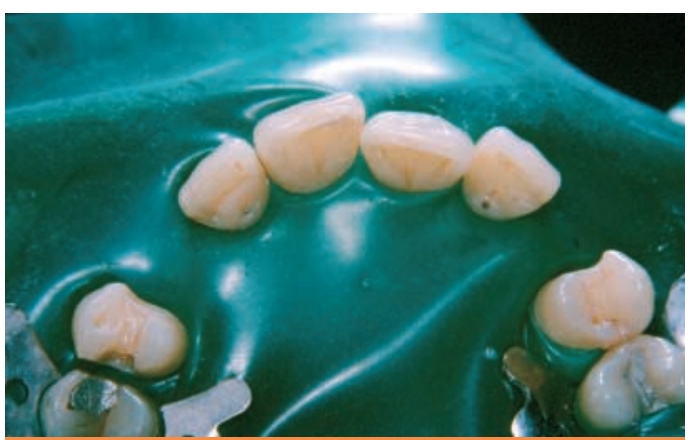

Fig. 58 Vue clinique avant collage.

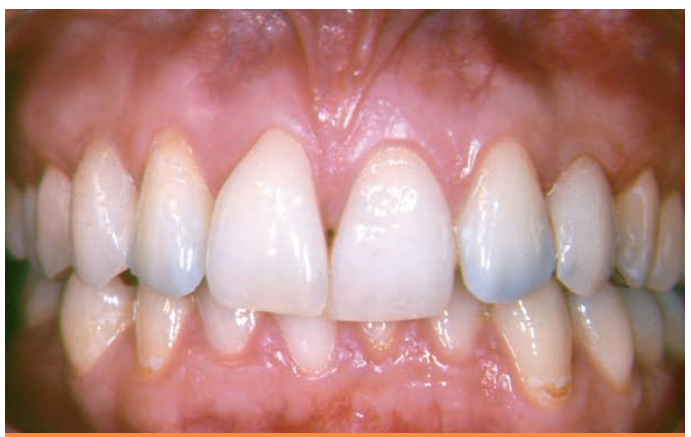

Fig. 60 Résultat clinique.

Réalisation prothétique : J. Ollier. 
Analyse de l'option implantaire :

l'implant en remplacement de la canine maxillaire

Lorsque le traitement est complexe, à savoir qu'il existe de grosses dysharmonies dentaires et squelettiques nécessitant une approche chirurgicale, cette phase sera mise à profit pour optimiser le volume osseux afin de permettre l'approche implantaire au niveau du site de la canine.
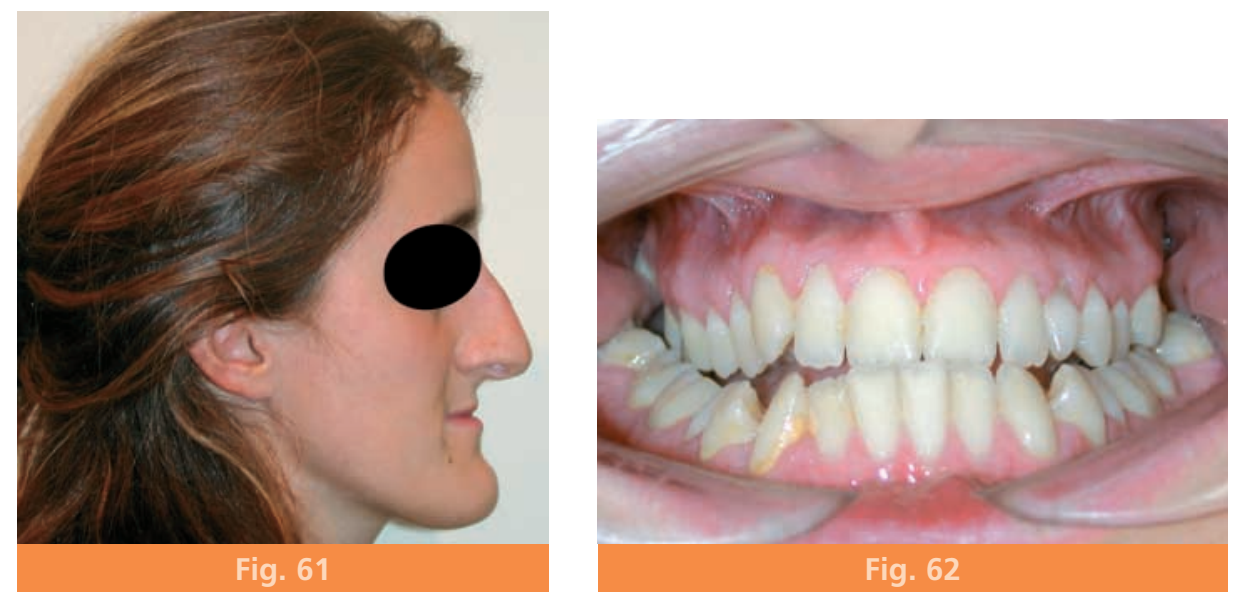

Fig. 61 et Fig. 62

Patiente affectée d'une importante Classe III. Le traitement a été à la fois orhodontique et chirurgical. Au cours d'une double ostéotomie maxillaire et mandibulaire associée à une génioplastie, un renforcement alvéolaire sera réalisé dans le site 23 .
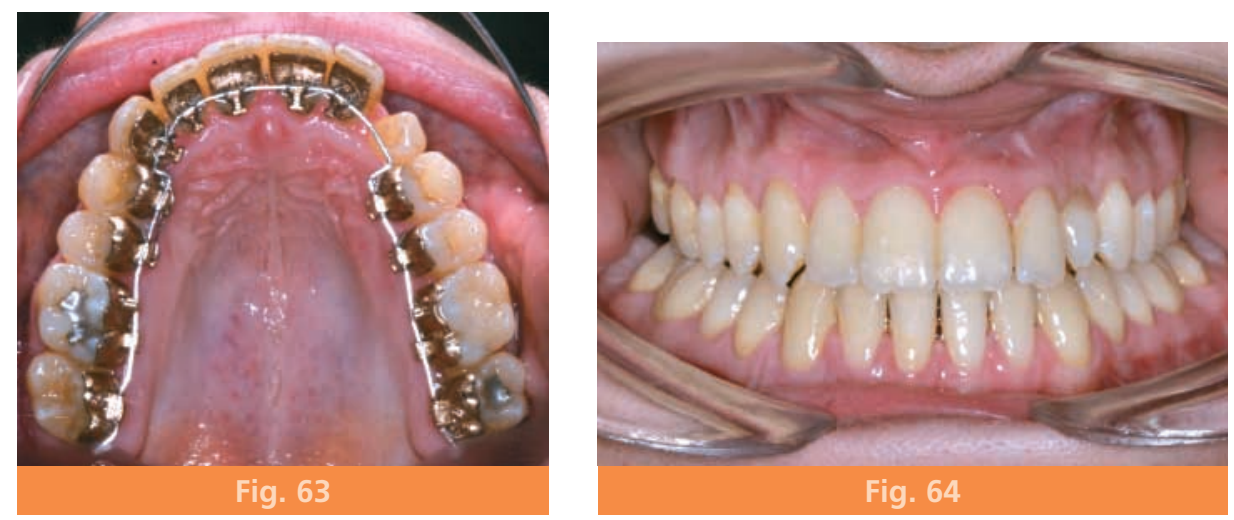

Fig. 63 et Fig. 64

Traitement orthodontique : Ephraim Medina. 

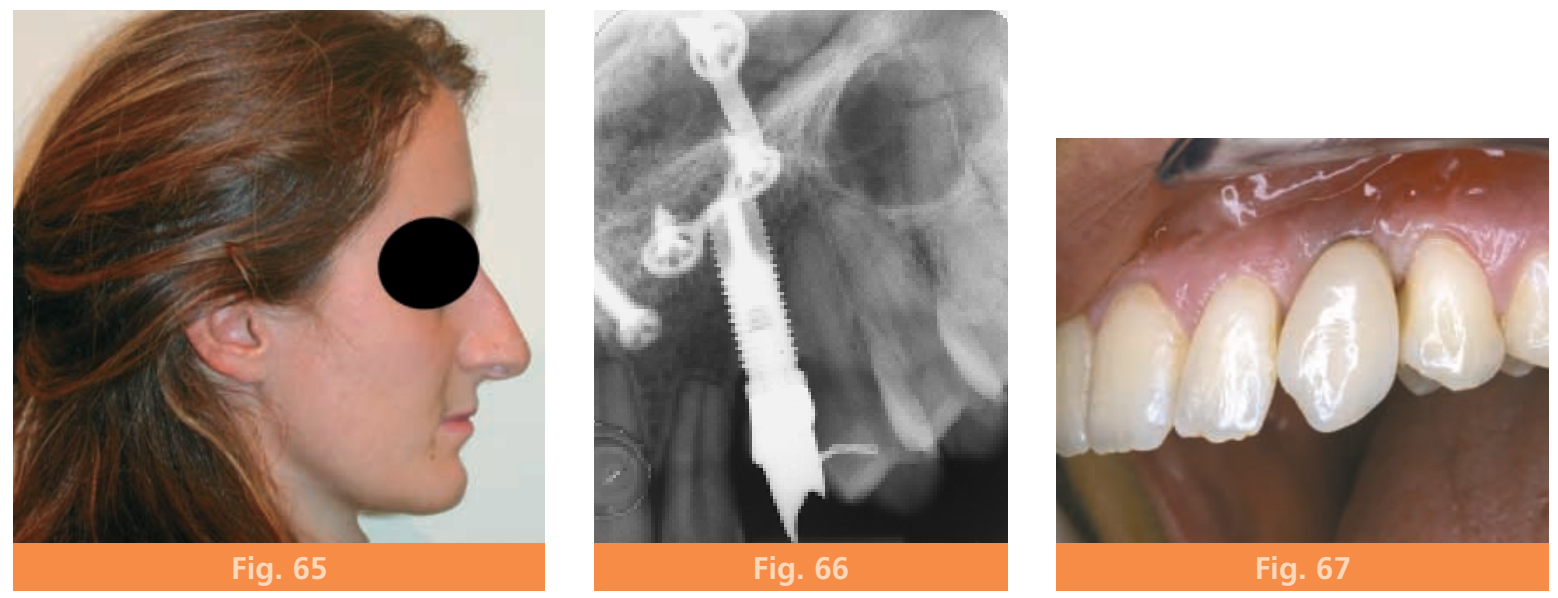

Fig. 65, Fig. 66 et Fig. 67

Résultat après traitement / Céramo-céramique sur pilier titane.

Traitement chirurgical : J.-F. Tulasne.

Traitement prothétique : F. Bouadi, Y. Samama, Laboratoire L. Coudray.

Implant en remplacement de la canine mandibulaire

À I'arcade inférieure, les conditions cliniques d'autre part des conséquences esthétiques sont souvent plus favorable compte tenu moins difficiles à contrôler.

d'une part de la qualité de l'os alvéolaire et
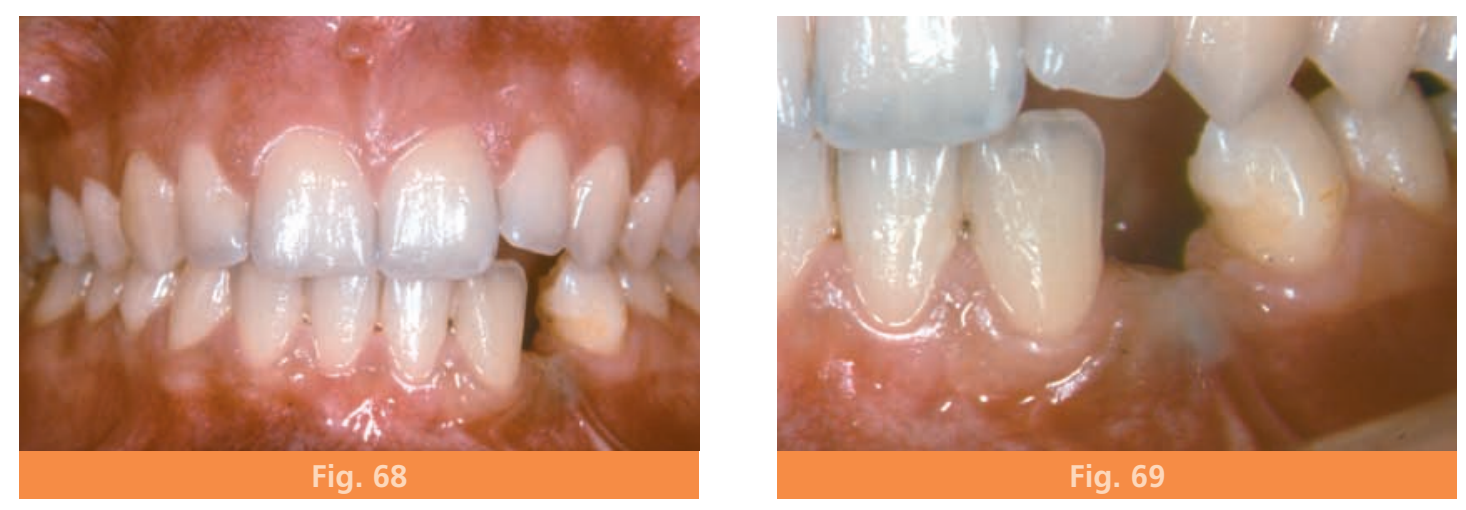

Fig. 68 et Fig. 69

Vue clinique préopératoire après traitement orthodontique destiné à créer un espace suffisant pour la pose d'un implant notamment en redressant la version de la prémolaire. 


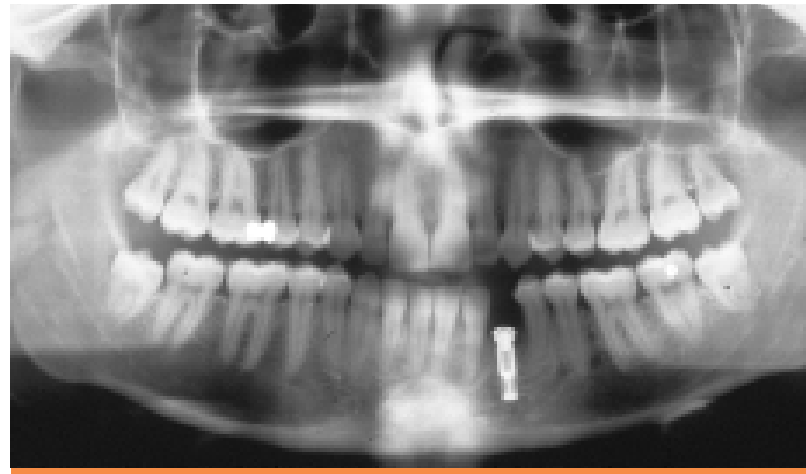

Fig. 70 Radio panoramique: atteste de la pose d'un implant.

Traitement chirurgical : J.-F. Tulasne.

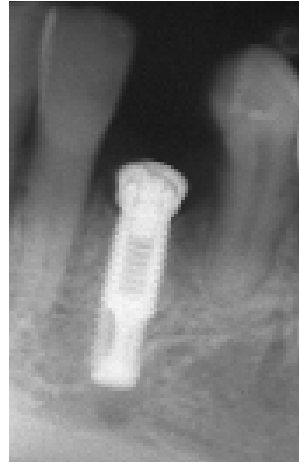

Fig. 71

Radio rétro alvéolaire de contrôle de l'implant posé.

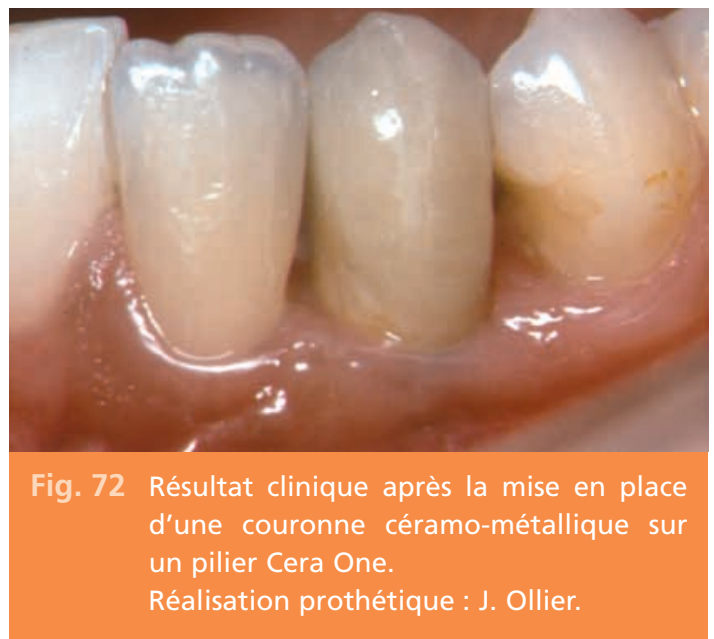

\section{Agénésie de la canine associée à d'autres agénésies}

L'agénésie de la canine maxillaire est souvent associée à des agénésies multiples. Dans le tableau II, on notera la fréquence de l'agéné- sie de la canine dans une population présentant des agénésies multiples[16, 17]. 
Tableau II

Prévalence des types de dents absentes chez des patients présentant des agénésies multiples[16, 17]. Ces chiffres sont obtenus à partir de 24 études où 11422 agénésies dentaires ont été recensées.

L'agénésie de la canine maxilaire arrive en 4 e position. On note que sur 11422 dents absentes, 22,9\% sont des incisives latérales. D'après la méta-analyse de Polder et coll.[16, 17].

\begin{tabular}{llccc}
\hline & \multicolumn{2}{c}{ Maxillaire } & \multicolumn{2}{c}{ Mandibule } \\
\hline I1 & $\mathrm{n}$ & Pourcentage & $\mathrm{n}$ & Pourcentage \\
\hline I2 & 18 & 0,2 & 403 & 3,5 \\
$\mathrm{C}$ & 2620 & 22,9 & 282 & 2,5 \\
P1 & 149 & 1,3 & 39 & 0,3 \\
P2 & 320 & 2,8 & 161 & 1,4 \\
M1 & 2423 & 21,8 & 4687 & 41,0 \\
M2 & 81 & 0,7 & 31 & 0,3 \\
Total & 67 & 0,6 & 141 & 1,2 \\
\hline
\end{tabular}
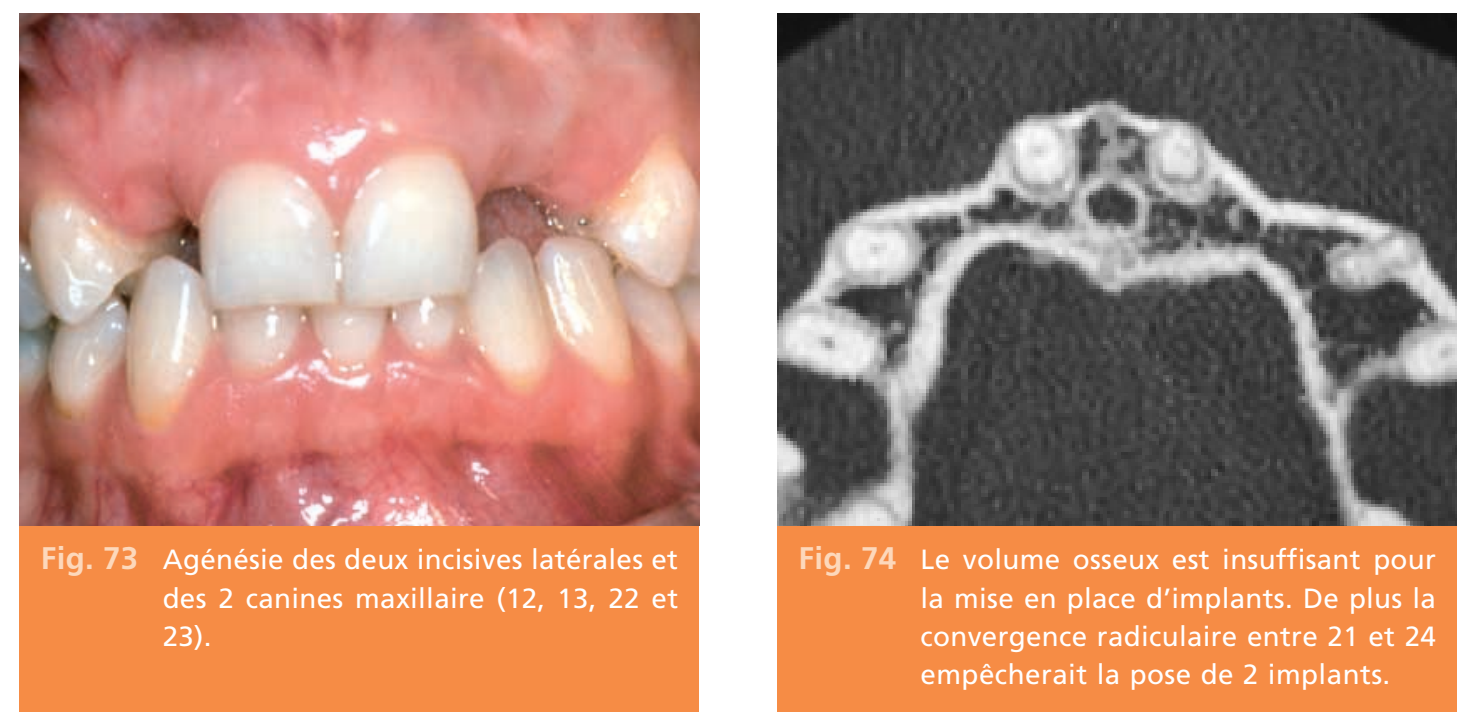

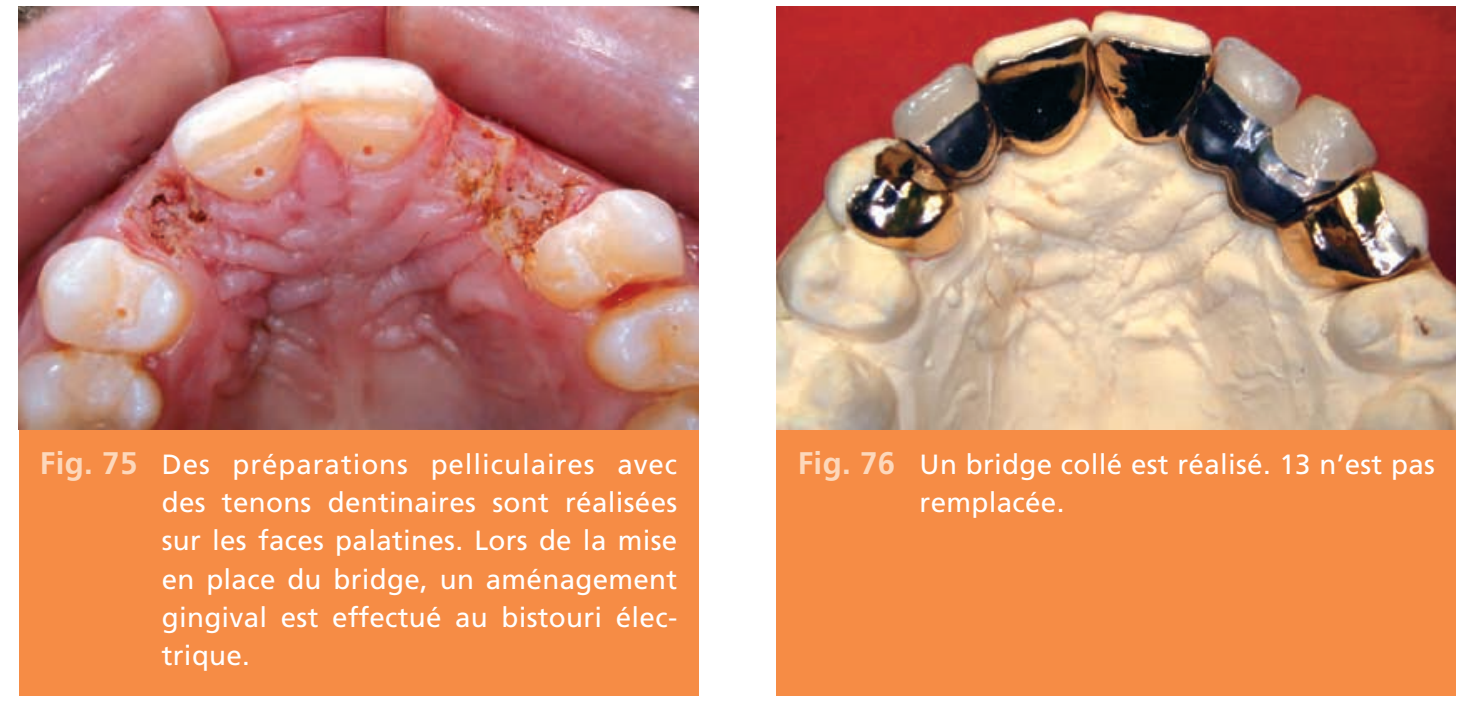

Fig. 76 Un bridge collé est réalisé. 13 n'est pas remplacée.

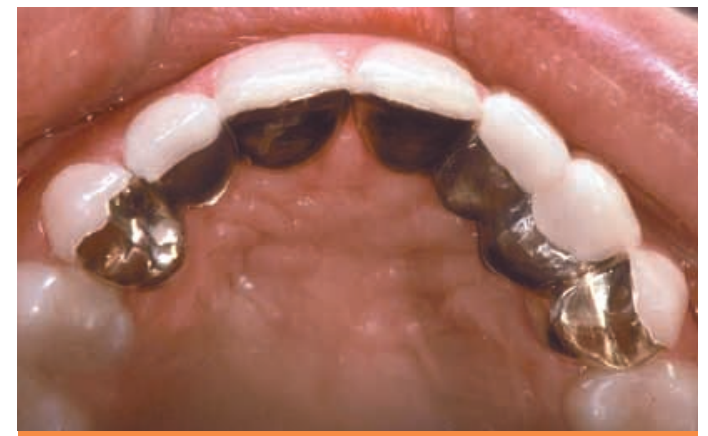

Fig. 77 Vue palatine de la construction après sa mise en place.

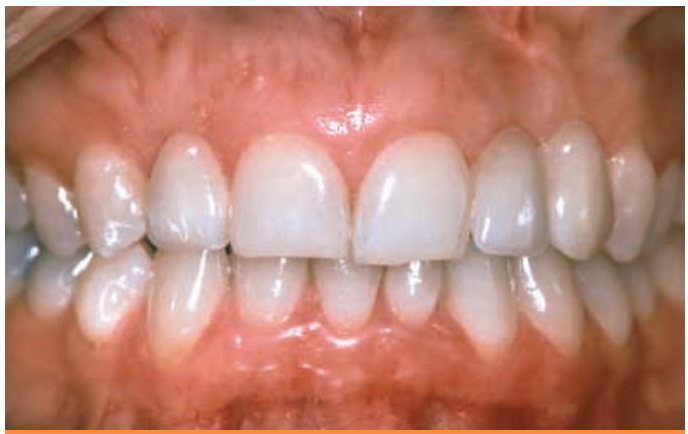

Fig. 78 Vue vestibulaire. La 14 est en position de 13 . II n'y a pas de préjudice esthétique.

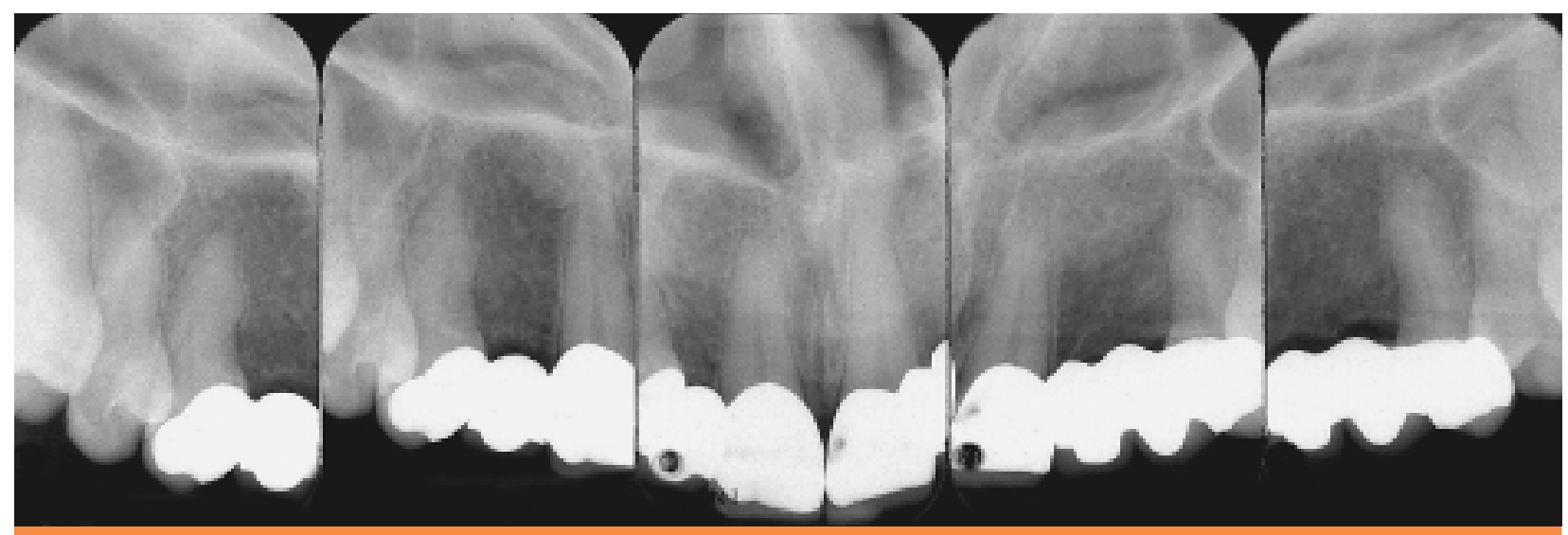

Fig. 79 Radio de contrôle de la construction 8 semaines post-opératoire. 


\section{Agénésie multiple bi-maxillaire}

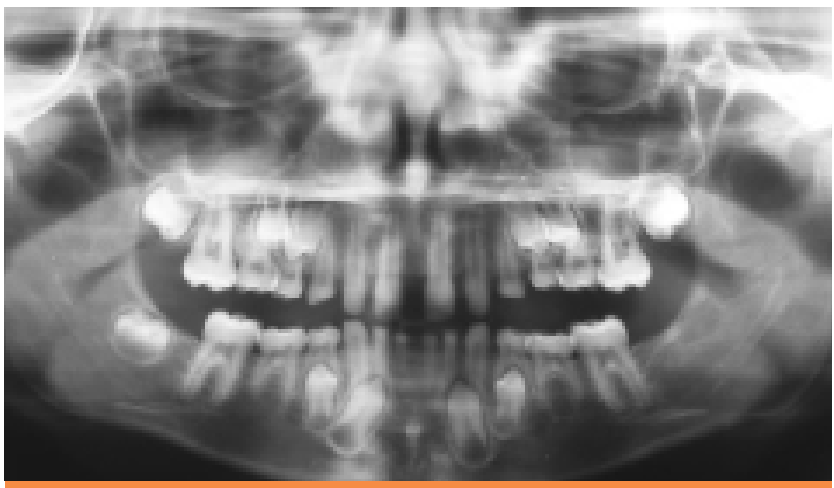

Fig. 80

Situation d'agénésies multiples:

- arcade mandibulaire : 41, 42, 45, 31, 32, 35, 37 ;

- arcade maxillaire : 13 et 23 : Les 2 canines maxillaires doivent être remplacées après le traitement orthodontique.

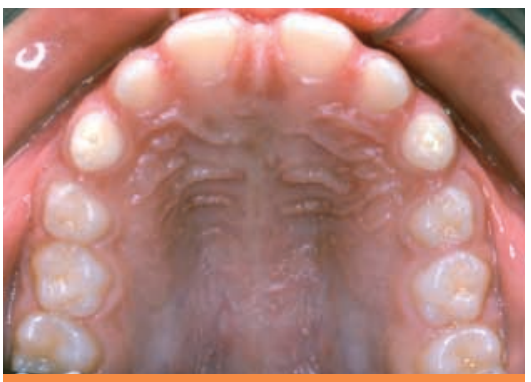

Fig. 81 Situation initiale en 1992.

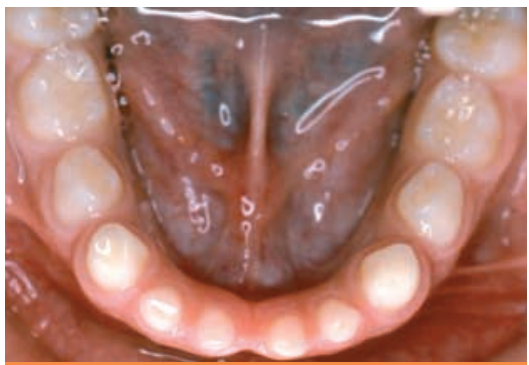

Fig. 82 Situation initiale en 1992.

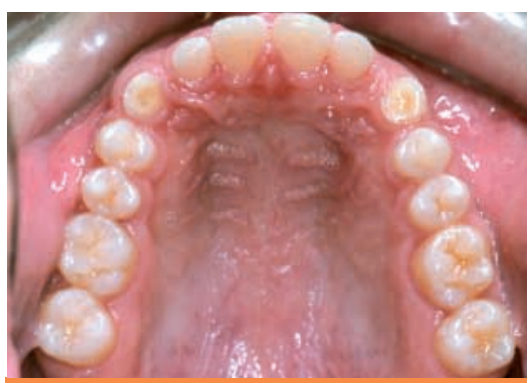

Fig. 84 Situation en 2000 après orthodontie.

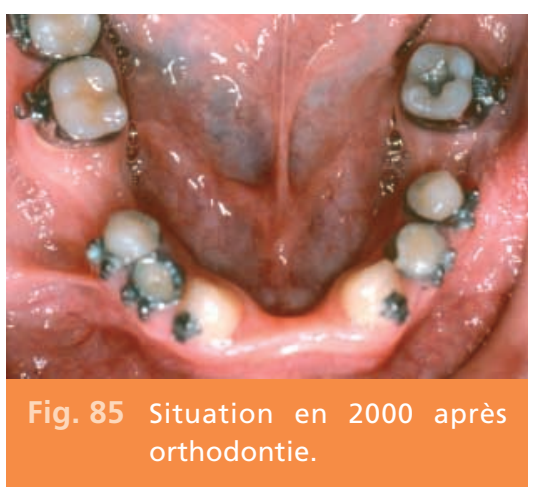

Actualités Odonto-Stomatologiques - $n^{\circ} 244$ - décembre 2008 


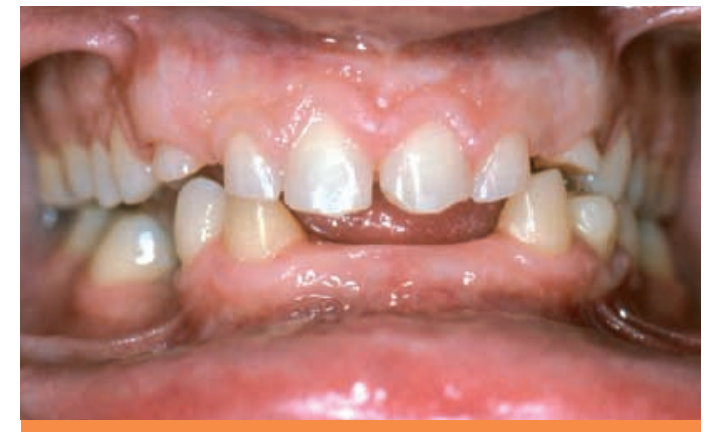

Fig. 86 Phase de finition du traitement orthodontique : on note qu'à l'arcade maxillaire les canines lactéales ont été gardée le plus longtemps possible afin de permettre le maintien de l'espace coronaire et de favoriser par leur résorption le volume osseux.

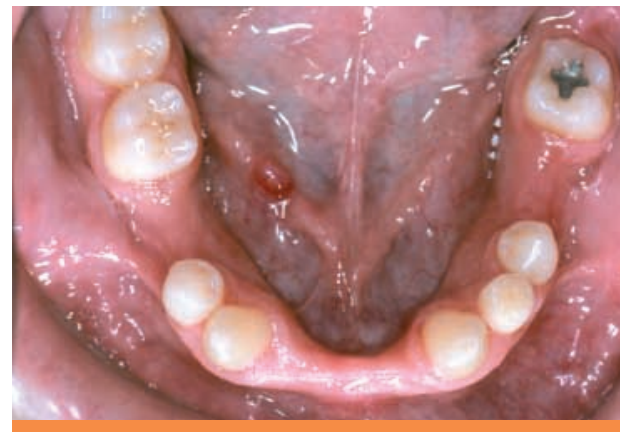

Fig. 872003

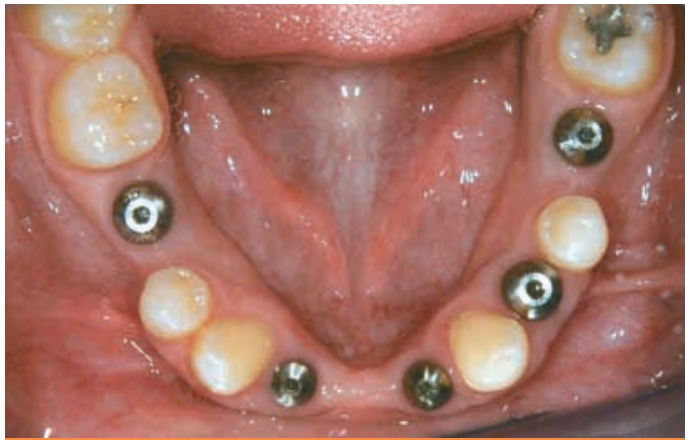

Fig. 89 A la mandibule 5 implants sont placés dans les secteurs édentés.

Traitement chirugicale : J.-F. Tulasne.

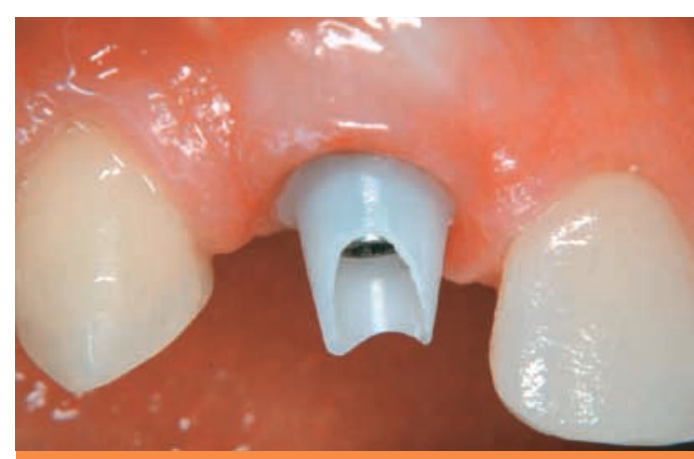

Fig. 90 L'utilisation de composants prothétiques en zircone sur des implants en situation de 13 et 23 permet d'optimiser le résultat esthétique final. 


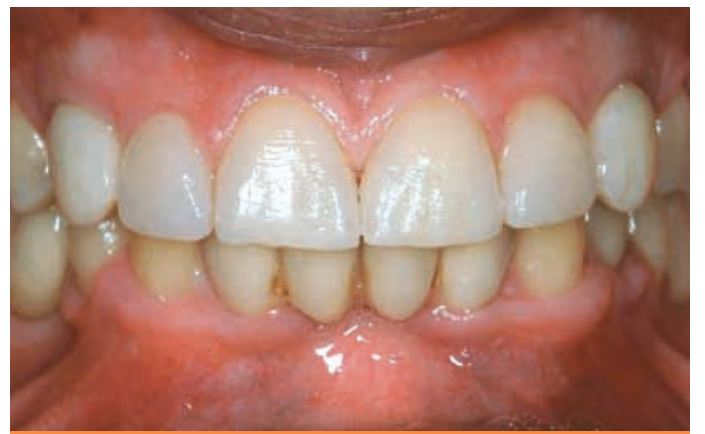

Fig. 91 Situation en 2004 après la pose de facettes sans préparation sur les incisives.

Réalisation prothétique : L. Coudray.

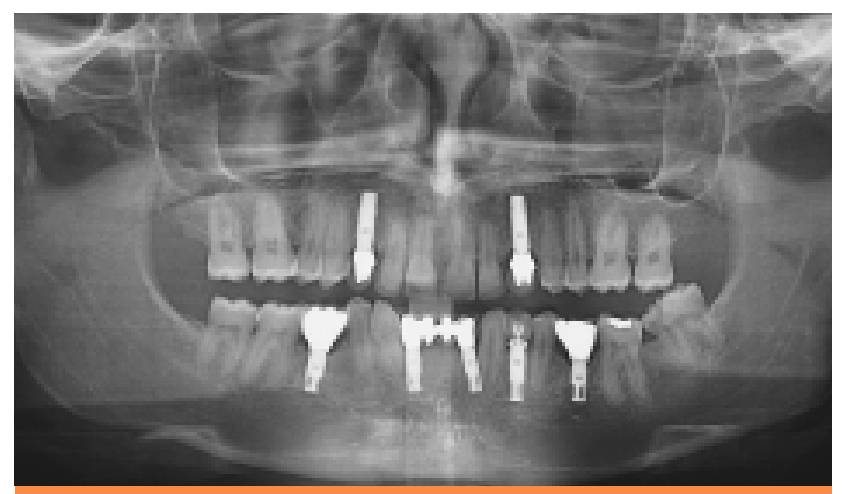

Fig. 92 Radio panoramique de contrôle 2 ans post-opératoire.
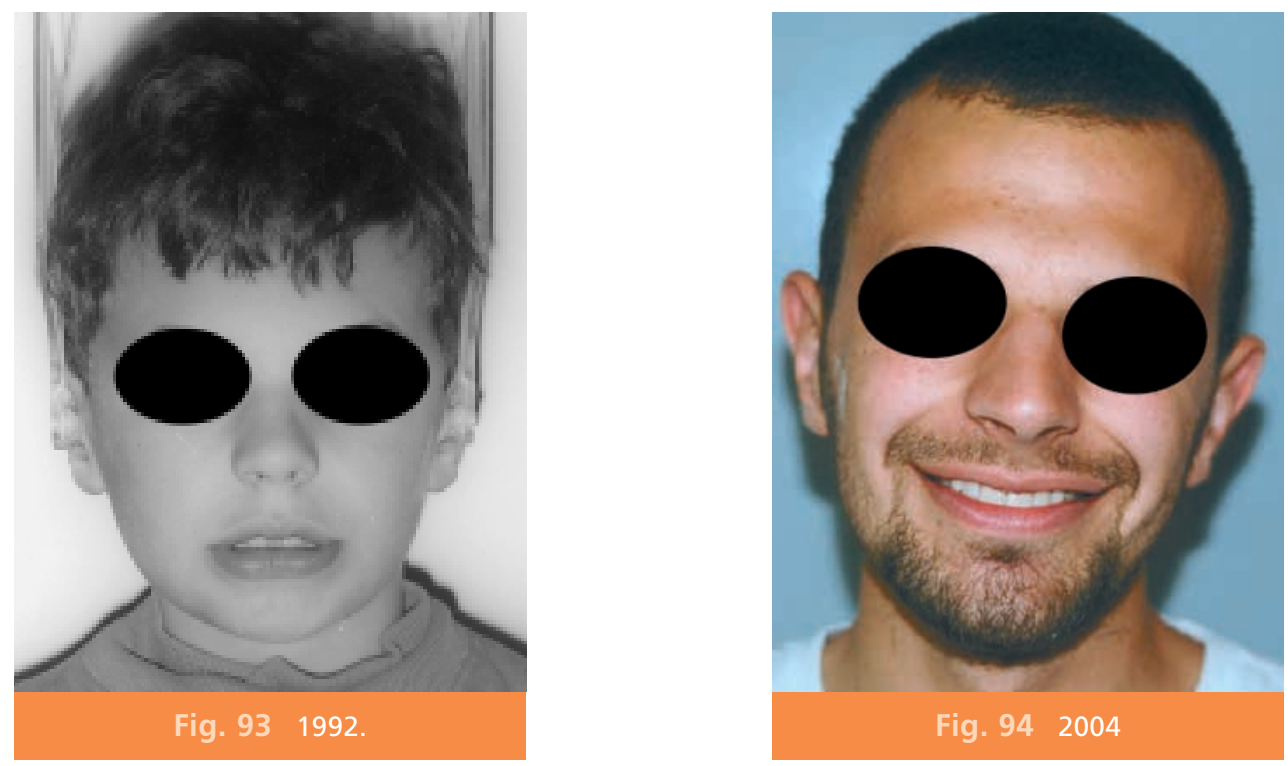


\section{Conclusion}

Le remplacement de la canine constitue un bon type de description quant aux problèmes posés par le remplacement de la dent unitaire en prothèse conjointe. En effet, au niveau de cette dent les impératifs fonctionnels et esthétiques sont majeurs et étroitement liés.

L'économie tissulaire constituera l'objectif essentiel tant au point de vue de la pérénité des solutions prothètiques qu'au plan biologique, et dans ce sens, I'implant touve toute sa valeur.

Cependant le concept de non mutilation des organes dentaires ne doit pas conduire à un nouveau dogme en systématisant des solutions types.

Des traitements purement prothétiques conservent des indications car chaque situation clinique est fonction de données objectives et subjectives. L'utilisation d'onlays comme moyen d'ancrage de bridge collé, apporte une réponse au cahier des charges posé par le remplacement de la canine maxillaire. Ces procédés permettent une économie tissulaire importante par rapport aux prépara- tions périphériques céramo-métalliques. En revanche, il faut souligner que les nouveaux matériaux, tel le zircone, qui permettent de s'affranchir du métal dans les procédés céramo-céramiques, ne donnent pas de résultants probants dans les techniques d'onlays moyens ancrage de bridges collés.

En outre, la notion de pluridisciplinarité doit constamment être présente à l'esprit car elle peut constituer une proposition efficace pour optimiser le replacement de cette dent à condition qu'elle ne soit pas dénaturée par une solution prothétique inapropriée.

En prothèse conjointe, la mise en opposition entre collage et implantologie constitue un abus de priorité fondé sur une appréciation trop précipitée de la notion d'épreuve du temps. L'indication du choix prothétique pour le remplacement de la canine devra toujours être nuancé en fonction des conditions de faisabilité, à travers l'évaluation des objectifs du praticien et des besoins du patient, du moment d'interception, du rendement esthétique et fonctionnel réel.

\section{Bibliographie}

1. Rochette Al. Attachement of a splint to enamel of the lower anterior teeth. J. Prosthet Dent 1973;30: 418-423.

2. Brabant A. Les clés du succès des bridges collés : indication

et forme de contour.

Rev. d'odontostomatologie 1995;24;6:489-497.

3. Degrange M, Bouter D. Factors influencing the reliability of resin-bonded fixed partial dentures. In «Minimally invasive restorations with bonding" Degrange $M$ et Roulet J.F. Quintessence Publishing Co, Inc 1997:153-176.

4. Samama $Y$, Fontenelle $A$, Nguyen $\mathrm{T}$. 
Analyse des options prothétiques dites «non-invasives» dans le traitement des agénésies isolées. Type de description : I'agénèsie uniou bilatérale de l'incisive latérale maxillaire.

Journal de Parodontologie 1993;12(1):73-82.

5. Samama Y. Fixed bonded prosthodontics: a 10-year follow-up report.

Part I:

analytical overview. Int J Periodontics Restorative Dent 1995;15(5):424-435.

6. Samama $Y$.

Fixed bonded prosthodontics: a 10-year follow-up report.

Part II: Clinica assessment. Int J Periodontics Restorative Dent 1996;16(1):52-59.

7. Samama $Y$, Nguyen $T$, Attal J.P.

Prothèse collée prothèse sur implant : les critères de choix. Rev. d'odontostomatologie 1995:24(6):549-572.

8. Adell $R$, Lekholm $U$, Rockler B, Branemark PI. A 15-year study of osseointegrated imlants in the treatment of the edentulous jaw. Int J Oral Surg 1981;10: 387-410.

9. Branemark PI, Zarb G, Albrektsson T (eds).

Tissu integrated prostheses.

Osseointegration in clinical dentistry. Chicago : Quintessence, 1985.

10. Jemt $\mathrm{T}$, Laney $\mathrm{W}$, Haris $\mathrm{D}$. Osseointegrated implants for single tooth replacement: A 1 year report from a multicenter prospective study. Int J Oral

Maxillofac Implants 1991;6:26-36.

11. Haas $R$,

Mensdorff-Pouilly N,

Mailath G, Watzek G.

Branemark

single tooth implants:

a preliminary report

of 76 implants.

J Prosthet Dent 1995; 73(3):247-279.

12. Henry PJ, Laney WR, Jemt T, Harris D, Krogh PH, Polizzi G et al.

Osseointegrated implants for single tooth replacement: a prospective 5 year multicenter study. Int J Oral

Maxillofac Implants 1996;11(4):450-455.
13. Priest GF.

Single tooth implants and their role

in preserving remaining teeth: a 10-year survival study. Int J Oral

Maxillofac Implants 1999 mar-apr;14(2):181-188.

14. Scheller $\mathrm{H}$, Urgell JP, Kultje C, Klineberg I, Goldberg PV, Stevenson-Moore $\mathrm{P}$ et al. A 5 year multicenter study on implant-supported single crown restorations. Int J Oral

Maxillofac Implants 1998;13(2):212-218.

15. Gardella JP. Communication au Symposium d'Implantologie de Porto Veccio. 2007.

16. Polder BJ, Van't Hof MA, Van der Linden FP Kuijpers - Jagtman AM. A méta-analysis of the prevalence of dental agenesis of permanent teeth. Community Dent Oral Epidemiol 2004; 32(3):217-226.

17. Marc Thierry, Jean Granat, Laurent Vermelin. Les agénésies dentaires: origine, évolution et orientation thérapeutiques. International Orthodontics 2007;5:163-182. 


\section{SUMMARY}

\section{The canine remplacement, analysis and case reports}

Yves SAMAMA

Philippe RAJZBAUM

Keywords

- agenesis

- canine

- included canine

- resin bonded bridge

- implant
Two elements have led us to analyse the latest treatment possibilities regarding prosthetic therapies in cases of canine absence; firstly, the possibility today of earlier detection of agenesis or included canines and secondly, the current aesthetic complaints of untreated elderly patients. Analysis between bonded bridges and single tooth implant constructions has been undertaken.

This article points out that apart from the usual analysis of the clinical criteria necessary in deciding any prosthetic work, it is also useful to take into account the age and motivation of the patient with regards to the length and difficulty of the treatment in question. Implantation can not systematically be favoured in every case and a fixed prosthesis would often seem to the advisable solution.

The difficulty in evaluating aesthetic problems connected with the implantsupported prosthesis has often led to disappointments or failures. To our mind, such difficulties often seem related to a poor understanding of the various problems concerning different types of tooth loss, each involving differing levels of tissue damage. In situations where initial conditions are unfavourable to an implant approach (limited coronal space, extensive osteomucosa defects in patients with a poly-traumatic condition) the efforts undertaken may sometimes appear over exaggerated; it is interesting to consider the ease with which fixed prosthetic restorations are carried out in certain cases.

Les auteurs remercient Laurie Arnaud pour l'aide apportée à la rédaction de cet article. 